\title{
Prediction of microalbuminuria from proteinuria in chronic kidney disease due to non-diabetic lifestyle-related diseases: comparison with diabetes
}

\author{
Makoto Ogi ${ }^{1} \cdot$ Takuya Seto $^{1} \cdot$ Yoshinori Wakabayashi $^{1}$
}

Received: 31 August 2020 / Accepted: 28 January 2021 / Published online: 3 March 2021

(c) The Author(s) 2021

\begin{abstract}
Background To suppress increases in kidney failure and cardiovascular disease due to lifestyle-related diseases other than diabetes, early intervention is desirable. We examined whether microalbuminuria could be predicted from proteinuria.

Methods The participants consisted of adults who exhibited a urinary protein-to-creatinine ratio (uPCR) of $<0.5 \mathrm{~g} / \mathrm{gCr}$ and an eGFR of $\geq 15 \mathrm{ml} / \mathrm{min} / 1.73 \mathrm{~m}^{2}$ in their spot urine at their first examination for lifestyle-related disease. Urine was tested three times for each case, with microalbuminuria defined as a urinary albumin-to-creatinine ratio (uACR) of 30-299 mg/ $\mathrm{gCr}$, at least twice on three measurements. Youden's Index was used as an index of the cut-off value (CO) according to the ROC curve.

Results A single uPCR was useful for differentiating normoalbuminuria and micro- and macroalbuminuria in patients with non-diabetic lifestyle-related diseases. Regarding the GFR categories, the $\mathrm{CO}$ of the second uPCR was $0.09 \mathrm{~g} / \mathrm{gCr}$ (AUC 0.89 , sensitivity 0.76 , specificity 0.89 ) in G1-4 $(n=197)$ and $0.07 \mathrm{~g} / \mathrm{gCr}$ (AUC 0.92 , sensitivity 0.85 , specificity 0.88 ) in G1-3a $(n=125)$. Using the sum of two or three uPCR measurements was more useful than a single uPCR for differentiating microalbuminuria in non-diabetic lifestyle disease [CO, $0.16 \mathrm{~g} / \mathrm{gCr}$ (AUC 0.91, sensitivity 0.85 , specificity 0.87 ) and $0.23 \mathrm{~g} /$ $\mathrm{gCr}$ (AUC 0.92, sensitivity 0.88 , specificity 0.84 ), respectively].

Conclusion Microalbuminuria in Japanese individuals with non-diabetic lifestyle-related diseases can be predicted from the uPCR, wherein the $\mathrm{CO}$ of the uPCR that differentiates normoalbuminuria and micro- and macroalbuminuria was $0.07 \mathrm{~g} / \mathrm{gCr}$ for G1-3a, while that in G3b-4 was $0.09 \mathrm{~g} / \mathrm{gCr}$.
\end{abstract}

Keywords Lifestyle-related disease $\cdot$ Chronic kidney disease $\cdot$ Non-diabetes mellitus $\cdot$ Microalbuminuria $\cdot$ Proteinuria

\section{Introduction}

A GFR of $<60 \mathrm{ml} / \mathrm{min} / 1.73 \mathrm{~m}^{2}$ or a urinary albumin-to-creatinine ratio ( $\mathrm{uACR}$ ) of $\geq 30 \mathrm{mg} / \mathrm{gCr}$ is independent risk factor for all-cause mortality, cardiovascular mortality, kidney failure, acute kidney injury, and kidney disease progression in chronic kidney disease (CKD) [1, 2]. In Japan, diabetic nephropathy is the most common primary disease among the prevalent dialysis patients, and the rate of nephrosclerosis due to lifestyle-related diseases other than diabetes has been

Makoto Ogi

ogi@yuurinkouseikai.or.jp

1 Department of Internal Medicine, Yuurinkouseikai Fuji Hospital, 1784 Niihashi, Gotemba, Shizuoka 412-0043, Japan increasing [3], making it necessary to prevent kidney failure by early intervention for microalbuminuria, as albuminuria is a risk factor for CKD progression and kidney failure [1,2, 4]. Microalbuminuria is also a risk factor for cardiovascular diseases and death in lifestyle-related diseases [5-7], such as diabetes [4], hypertension [8], and metabolic syndrome [9], as well as in the general population [10]. Furthermore, albuminuria is a risk factor for the development of Alzheimer's disease and vascular dementia [11], which are common reasons for individuals requiring care in an aging society.

CKD is defined as an abnormal kidney structure and function, which persist for more than 3 months in KDIGO [1]. One marker of kidney damage is albuminuria $\geq 30 \mathrm{mg} / 24 \mathrm{~h}$ or $\mathrm{ACR} \geq 30 \mathrm{mg} / \mathrm{gCr}$ [1]. CKD is classified based on cause, GFR, and albuminuria category, and the boundary between A1 (normal to mildly increased) and A2 (moderately increased) is albuminuria $30 \mathrm{mg} / 24 \mathrm{~h}$ or uACR $30 \mathrm{mg} /$ 
$\mathrm{gCr}$ for all primary diseases [1]. Proteinuria, with a urinary protein-to-creatinine ratio (uPCR) of $150-500 \mathrm{mg} / \mathrm{gCr}$ is considered to be equivalent to A2 albuminuria (30-300 mg/ $\mathrm{gCr}$ ) [1]. On the other hand, in CKD staging in Japan, when the primary disease is diabetes, the boundary between $\mathrm{A} 1$ and $\mathrm{A} 2$ is a uACR of $30 \mathrm{mg} / \mathrm{gCr}$; however, in CKD due to non-diabetic lifestyle-related diseases, the boundary between $\mathrm{A} 1$ and $\mathrm{A} 2$ is a uPCR of $0.15 \mathrm{~g} / \mathrm{gCr}$ [2].

While dipstick proteinuria is a risk factor for kidney failure [12], along with cardiovascular disease and death, albuminuria is a superior predictor of cardiovascular disease [13]; thus, a method for predicting microalbuminuria is desired. However, the dipstick method cannot adequately evaluate small amounts of albuminuria [14]. Although there have been reports on the relationship between proteinuria and albuminuria [14-20], many reports indicate that it is difficult or inaccurate to predict microalbuminuria based on proteinuria $[14,17,19]$. It has been reported that the uPCR and $\mathrm{UACR}$ show a similar association with the risk of kidney disease progression in children with CKD without diabetes [20]. However, there appear to be no sufficient studies on whether a uPCR of $0.15 \mathrm{~g} / \mathrm{gCr}$ is optimal as a boundary value for detecting patients with a uACR of $\geq 30 \mathrm{mg} / \mathrm{gCr}$ among adults with non-diabetic lifestyle-related diseases in Japan.

The objective of this study was to investigate whether proteinuria could predict albuminuria of $<300 \mathrm{mg} / \mathrm{gCr}$, including a uACR of $30 \mathrm{mg} / \mathrm{gCr}$, which is the boundary value for $\mathrm{A} 1$ and 2 in non-diabetic lifestyle-related diseases. We also aimed to compare the relationship to that in patients with diabetes.

\section{Methods}

The subjects included Japanese patients of $\geq 18$ years of age who visited the Yuurinkousekusei Fuji Hospital during the period from October 2017 to September 2019 for lifestylerelated diseases or CKD due to the diseases or aging, with an eGFR of $\geq 15 \mathrm{ml} / \mathrm{min} / 1.73 \mathrm{~m}^{2}$ and a uPCR of $<0.5 \mathrm{~g} / \mathrm{gCr}$ at initiation. Patients with urinary tract infection, nephritis, hereditary renal disease, paraproteinemia, or cancer were excluded. Patients who gave their written consent to participate in the study were chosen as subjects. A history of lifestyle-related diseases and smoking was noted and their blood pressure, BMI, waist circumference, blood glucose, $\mathrm{HbA1c}$, serum uric acid, LDL-C, triglycerides, and nonHDL cholesterol levels were measured or the results of a health checkup were noted. Proteinuria and urinary occult blood were detected using the dipstick method, and urinary sediment was performed. At the same time, urinary protein and creatinine were measured to calculate uPCR. eGFR was calculated from age, sex, and serum creatinine [2].
Albumin was measured in the same urine sample of patients with a uPCR of $<0.5 \mathrm{~g} / \mathrm{gCr}$ to determine the uACR. Proteinuria and albuminuria were measured three times on different days, including the first measurement, with the second and third measurements performed using early morning spot urine [1]. Patients whose proteinuria increased to $\geq 0.5 \mathrm{~g} / \mathrm{gCr}$ on the second or third measurement were not excluded. The patients visited the clinic up to once every 3 months, depending on their CKD stage, with the eGFR

Table 1 Characteristics of patients

\begin{tabular}{|c|c|c|c|c|c|}
\hline & \multicolumn{2}{|c|}{$\begin{array}{l}\text { Non-diabetes } \\
\text { mellitus }\end{array}$} & \multicolumn{2}{|c|}{ Diabetes mellitus } & \multirow[t]{2}{*}{$p$ value } \\
\hline & $(n=197)$ & $(\%)$ & $(n=106)$ & $(\%)$ & \\
\hline Age (years) & \multicolumn{2}{|l|}{$68.9 \pm 14.8$} & \multicolumn{2}{|l|}{$67.1 \pm 11.6$} & 0.85 \\
\hline $\operatorname{Sex}(m: f)$ & \multicolumn{2}{|l|}{$124: 73$} & \multicolumn{2}{|l|}{$70: 36$} & 0.59 \\
\hline Obesity & 61 & $(31.0)$ & 46 & (43.4) & 0.031 \\
\hline \multicolumn{6}{|c|}{ Waist circumference } \\
\hline Male $\geq 85 \mathrm{~cm}$ & 72 & $(58.1)$ & 49 & $(70.0)$ & 0.10 \\
\hline Female $\geq 90 \mathrm{~cm}$ & 22 & $(30.1)$ & 16 & $(44.4)$ & 0.14 \\
\hline \multicolumn{6}{|l|}{ Smoking } \\
\hline Previous & 59 & $(29.9)$ & 35 & $(33.0)$ & 0.58 \\
\hline Current & 22 & $(11.2)$ & 13 & $(12.3)$ & 0.78 \\
\hline \multicolumn{6}{|c|}{ Complicated disease } \\
\hline Hypertension & 126 & $(64.0)$ & 82 & $(77.4)$ & 0.017 \\
\hline Dyslipidemia & 113 & $(57.4)$ & 64 & $(60.4)$ & 0.61 \\
\hline Hyperuricemia & 86 & $(43.7)$ & 23 & $(21.7)$ & 0.00015 \\
\hline \multicolumn{6}{|c|}{ Dipstick proteinuria } \\
\hline$(-)$ & 143 & $(72.6)$ & 81 & $(76.4)$ & 0.47 \\
\hline$( \pm)$ & 30 & $(15.2)$ & 13 & $(12.3)$ & 0.48 \\
\hline $1(+)$ & 21 & $(10.7)$ & 11 & (10.4) & 0.94 \\
\hline $2(+)$ & 3 & $(1.5)$ & 1 & $(0.9)$ & 0.67 \\
\hline \multicolumn{6}{|l|}{ Gfr category } \\
\hline G1 & 5 & $(2.5)$ & 12 & $(11.3)$ & 0.0015 \\
\hline G2 & 47 & $(23.9)$ & 45 & $(42.5)$ & 0.00079 \\
\hline G3a & 73 & $(37.1)$ & 26 & $(24.5)$ & 0.026 \\
\hline G3b & 44 & $(22.3)$ & 16 & $(15.1)$ & 0.13 \\
\hline G4 & 28 & $(14.2)$ & 7 & $(6.6)$ & 0.048 \\
\hline \multicolumn{6}{|c|}{ Albuminuria category } \\
\hline $\begin{array}{l}\text { Normoalbumi- } \\
\text { nuria }\end{array}$ & 93 & $(47.2)$ & 51 & $(48.1)$ & 0.88 \\
\hline $\begin{array}{l}\text { Microalbumi- } \\
\text { nuria }\end{array}$ & 98 & $(49.7)$ & 55 & $(51.9)$ & 0.72 \\
\hline $\begin{array}{l}\text { Macroalbumi- } \\
\text { nuria }\end{array}$ & 6 & $(3.0)$ & 0 & & 0.07 \\
\hline \multicolumn{6}{|c|}{ Time between mesurements (days) } \\
\hline $1-2$ & $84.4 \pm 60.8$ & & $75.1 \pm 56.5$ & & 0.97 \\
\hline $2-3$ & $75.0 \pm 61.8$ & & $66.2 \pm 45.8$ & & 0.79 \\
\hline
\end{tabular}

Microalbuminuria: urinary albumin-to-creatinine ratio 30-299 mg/ $\mathrm{gCr}$ at least twice for 3 measurements. Data are shown as the mean \pm standard deviation

$C K D$ chronic kidney disease; GFR glomerular filtration rate 
calculated on each visit. Patients whose eGFR decreased by $\geq 30 \%$ within 3 months were excluded.

Microalbuminuria was defined as a uACR of 30-299 mg/ $\mathrm{gCr}$ on at least two of three measurements, while normoalbuminuria was defined as a uACR of $<30 \mathrm{mg} / \mathrm{gCr}$ on at least two of three measurements, and macroalbuminuria was defined as a uACR of $\geq 300 \mathrm{mg} / \mathrm{gCr}$ on at least two of three measurements. When uACR was $<30 \mathrm{mg} / \mathrm{gCr}, 30-299 \mathrm{mg} /$ $\mathrm{gCr}$, and $\geq 300 \mathrm{mg} / \mathrm{gCr}$, once each, then this condition was defined as microalbuminuria in this study. The average eGFR during the period was used to determine the GFR category in each case.

Urinary protein was measured using the pyrogallol red method (AR WAKO microTP-AR, Wako Pure Chemical Industries), urinary albumin was measured using an immunoturbimetric method (AutoWako Microalbumin, Wako Pure Chemical Industries), and serum and urine creatinine were measured using the enzyme method (L type WAKO CREM, FUJIFILM Wako Pure Chemical Corporation) with an autoanalyzer (TBA-2000FR).

Student's $t$ test and the chi-squared test were used to compare clinical symptoms, and the Mann-Whitney U test, and Kruskal-Wallis and Steel-Dwass tests were used to compare the urinary protein, urinary albumin, urinary creatinine, uACR, and uPCR values for each category. The median uACR and IQR ranging from uPCR $0.01 \mathrm{~g} / \mathrm{gCr}$ to $0.16 \mathrm{~g} / \mathrm{gCr}$ were examined for each uPCR $0.01 \mathrm{~g} / \mathrm{gCr}$ in all cases, non-diabetics and diabetics, respectively. The distributions of $\mathrm{UACR}$ and $\mathrm{uPCR}$ were found to be highly skewed (skewness test for normality, $\mathrm{p}<0.0001$ ); thus, the uACR and $\mathrm{UPCR}$ were log-transformed. The relationship between $\log$ uPCR and $\log$ median UACR was analyzed by a linear spline. The change in slope within the range between knots was investigated. We also analyzed the nonlinear association using a restricted cubic spline. Akaike's information criterion (AIC) was used for the data-based choice of the number of knots, and the knots were placed at standard locations [21].

The receiver operating characteristic (ROC) curve was used to determine whether a uACR of $\geq 30 \mathrm{mg} / \mathrm{gCr}$ and microalbuminuria could be differentiated by a single uPCR or a sum of two or three uPCRs and a single uACR, to obtain the cut-off value (CO), sensitivity ( $\mathrm{Sn}$ ), and specificity (Sp), with the largest Youden's index (YI) value, using the area under the curve (AUC). If the YI was the same, the $\mathrm{CO}$ with the larger $\mathrm{Sn}$ was adopted. The

Table $2 \mathrm{uACR}$, uPCR and uPCR/uACR in patients with and without diabetes mellitus according to GFR category

\begin{tabular}{|c|c|c|c|c|c|c|}
\hline GFR category & $\mathrm{n}$ & uACR (mg/gCr) & uACR undetected & uPCR (g/gCr) & uPCR undetected & $\begin{array}{l}\mathrm{uPCR} / \mathrm{uACR} \\
(\mathrm{g} / \mathrm{gCr}) /(\mathrm{g} / \\
\mathrm{gCr})\end{array}$ \\
\hline \multicolumn{7}{|c|}{ Non-diabetes mellitus } \\
\hline G1 & 15 & $48.2 \pm 29.8^{*}$ & 0 & $0.076 \pm 0.057$ & $3(20.0)$ & $1.53 \pm 0.94 *$ \\
\hline $\mathrm{G} 2$ & 141 & $71.6 \pm 95.8$ & $1(0.7)$ & $0.112 \pm 0.124$ & $9(6.4)$ & $2.86 \pm 3.76^{\mathrm{d}}$ \\
\hline G3a & 219 & $55.8 \pm 75.9$ & 0 & $0.102 \pm 0.117$ & $19(8.3)$ & $2.91 \pm 3.12$ \\
\hline G3b & 132 & $74.4 \pm 82.4$ & 0 & $0.120 \pm 0.116^{*}$ & $8(6.1)$ & $2.51 \pm 2.47$ \\
\hline G4 & 84 & $77.0 \pm 114.3^{* *}$ & 0 & $0.150 \pm 0.198 \mathrm{c} 1 *$ & $5(6.0)$ & $3.14 \pm 3.15^{*}$ \\
\hline GFR category & $\mathrm{n}$ & uACR (mg/gCr) & uACR undetected & uPCR (mg/gCr) & uPCR undetected & $\begin{array}{l}\mathrm{uPCR} / \mathrm{uACR} \\
(\mathrm{g} / \mathrm{gCr}) /(\mathrm{g} / \\
\mathrm{gCr})\end{array}$ \\
\hline \multicolumn{7}{|c|}{ Diabetes mellitus } \\
\hline G1 & 36 & $31.6 \pm 28.6$ & 0 & $0.072 \pm 0.075$ & $2(5.6)$ & $3.04 \pm 2.60$ \\
\hline $\mathrm{G} 2$ & 135 & $37.4 \pm 35.3$ & 0 & $0.074 \pm 0.064$ & $11(8.1)$ & $2.67 \pm 2.05$ \\
\hline $\mathrm{G} 3 \mathrm{a}$ & 78 & $52.7 \pm 62.8$ & 0 & $0.103 \pm 0.105$ & $5(5.7)$ & $2.56 \pm 2.04$ \\
\hline $\mathrm{G} 3 \mathrm{~b}$ & 48 & $87.6 \pm 83.4^{\mathrm{abc} 1}$ & 0 & $0.168 \pm 0.142^{\mathrm{abcl}}$ & 0 & $2.44 \pm 1.56$ \\
\hline G4 & 21 & $125.5 \pm 97.3^{\mathrm{abc} 2}$ & 0 & $0.180 \pm 0.119^{\mathrm{abc} 2}$ & 0 & $1.91 \pm 1.12$ \\
\hline
\end{tabular}

Data are shown as the mean \pm standard deviation. Undetected $\mathrm{uACR}$ and $\mathrm{uPCR}$ were regarded as $0 \mathrm{mg} / \mathrm{gCr}$ and $0 / \mathrm{gCr}$, respectively

$u A C R$ urinary albumin-to-creatinine ratio; uPCR, urinary protein-to-creatinine ratio; GFR, glomerular filtration rate

${ }^{*} p<0.05, * * p<0.01$ vs. diabetic patients by the Mann-Whitney's U test

${ }^{\mathrm{a}} p<0.01$ vs. G1

${ }^{\mathrm{b}} p<0.01$ vs. G2

${ }^{\mathrm{c} 1} p<0.05$ vs. C3a

${ }^{\mathrm{c} 2} p<0.01$ vs. C3a by the Steel-Dwass test

${ }^{\mathrm{d}}$ One patient with undetected albuminuria was excluded 
Table 3 Association of uPCR $(\mathrm{g} / \mathrm{gCr})$, the median uACR in patients with lifestyle-related disease

\begin{tabular}{|c|c|c|c|c|c|c|c|c|c|}
\hline \multirow[b]{2}{*}{ uPCR (g/gCr) } & \multicolumn{3}{|c|}{ All patients } & \multicolumn{3}{|c|}{ Non-diabetes mellitus } & \multicolumn{3}{|c|}{ Diabetes mellitus } \\
\hline & $n$ & $\begin{array}{l}\text { median } \\
\text { uACR (mg/ } \\
\mathrm{gCr})\end{array}$ & IQR & $n$ & $\begin{array}{l}\text { median } \\
\text { uACR (mg/ } \\
\mathrm{gCr})\end{array}$ & IQR & $n$ & $\begin{array}{l}\text { median } \\
\text { uACR }(\mathrm{mg} / \\
\mathrm{gCr})\end{array}$ & IQR \\
\hline 0.01 & 41 & 8.0 & $6.0-15.0$ & 24 & 8.0 & $5.8-12.5$ & 17 & 8.0 & $6.0-20.0$ \\
\hline 0.02 & 58 & 10.5 & $7.0-15.0$ & 35 & 8.0 & $6.0-15.5$ & 23 & 12.0 & $8.5-13.5$ \\
\hline 0.03 & 67 & 11.0 & $7.0-17.0$ & 42 & 12.0 & $8.3-17.0$ & 25 & 11.0 & $6.0-18.0$ \\
\hline 0.04 & 64 & 16.0 & $10.0-28.0$ & 39 & 19.0 & $9.0-32.0$ & 25 & 12.0 & $10.0-27.0$ \\
\hline 0.05 & 58 & 17.5 & $10.3-29.0$ & 35 & 18.0 & $10.5-27.0$ & 23 & 17.0 & $11.0-27.5$ \\
\hline 0.06 & 65 & 21.0 & $12.0-37.0$ & 43 & 19.0 & $11.5-36.5$ & 22 & 25.0 & $14.0-36.8$ \\
\hline 0.07 & 56 & 29.5 & $13.0-41.0$ & 37 & 28.0 & $12.0-35.0$ & 19 & 41.0 & $25.0-47.0$ \\
\hline 0.08 & 36 & 26.0 & $18.0-53.3$ & 25 & 25.0 & $14.0-53.0$ & 11 & 32.0 & $22.0-45.5$ \\
\hline 0.09 & 40 & 39.0 & $24.8-57.3$ & 27 & 43.0 & $25.0-57.5$ & 13 & 32.0 & $23.0-43.0$ \\
\hline 0.10 & 28 & 51.0 & $35.8-63.0$ & 22 & 48.0 & $36.5-58.8$ & 6 & 63.0 & $42.0-66.8$ \\
\hline 0.11 & 26 & 60.5 & $44.3-73.5$ & 16 & 68.0 & $58.3-77.3$ & 10 & 46.0 & $42.3-54.8$ \\
\hline 0.12 & 29 & 57.0 & $34.0-78.0$ & 18 & 59.0 & $46.3-79.3$ & 11 & 52.0 & $32.5-71.5$ \\
\hline 0.13 & 23 & 71.0 & $55.0-92.0$ & 13 & 79.0 & $55.0-101.0$ & 10 & 64.0 & $55.8-77.8$ \\
\hline 0.14 & 27 & 67.0 & $40.0-88.0$ & 18 & 78.5 & $39.0-104.3$ & 9 & 51.0 & $48.0-74.0$ \\
\hline 0.15 & 25 & 71.0 & $48.0-104.0$ & 18 & 72.5 & $50.3-104.8$ & 7 & 69.0 & $52.5-88.0$ \\
\hline 0.16 & 25 & 95.0 & $53.0-120.0$ & 13 & 116.0 & $79.0-133.0$ & 12 & 80.0 & $51.8-98.5$ \\
\hline
\end{tabular}

$u A C R$ urinary albumin-to-creatinine ratio; $u P C R$ urinary protein-to-creatinine ratio; $I Q R$ interquartile range

Table 4 Equations to estimate median uACR from uPCR using 4-knots linear spline and the change in slope before and after the range of the knots

\begin{tabular}{|c|c|c|c|c|c|c|c|c|c|c|c|c|c|c|c|}
\hline \multicolumn{2}{|c|}{ uPCR (mg/gCr) } & \multicolumn{3}{|c|}{$\begin{array}{l}\text { In patients with } \\
\text { lifestyle-related } \\
\text { disease }\end{array}$} & \multicolumn{4}{|c|}{ Non-diabetes and diabetes } & \multicolumn{4}{|c|}{ Non-diabetes } & \multicolumn{3}{|c|}{ Diabetes } \\
\hline \multirow{5}{*}{\multicolumn{2}{|c|}{$\ln ($ median uACR) }} & \multicolumn{3}{|c|}{$1 \mathrm{R} ;<20$} & \multicolumn{4}{|c|}{$0.3719 \times \ln (\mathrm{uPCR})+1.2232$} & \multicolumn{4}{|c|}{$0.2801 \times \ln (\mathrm{uPCR})+1.4345$} & \multicolumn{3}{|c|}{$0.2210 \times \ln (\mathrm{uPCR})+1.5706$} \\
\hline & & \multicolumn{3}{|c|}{$2 \mathrm{R} ; 20$ to $<60$} & \multicolumn{4}{|c|}{$0.6224 \times \ln (\mathrm{uPCR})+0.4727$} & \multicolumn{4}{|c|}{$0.6804 \times \ln (\mathrm{uPCR})+0.2352$} & \multicolumn{3}{|c|}{$0.8277 \times \ln (\mathrm{uPCR})-0.2471$} \\
\hline & & \multicolumn{3}{|c|}{$3 \mathrm{R} ; 60$ to $<120$} & \multicolumn{4}{|c|}{$1.5483 \times \ln (\mathrm{uPCR})-3.3183$} & \multicolumn{4}{|c|}{$1.6581 \times \ln (\mathrm{uPCR})-3.7678$} & \multicolumn{3}{|c|}{$1.1957 \times \ln (\mathrm{uPCR})-1.7535$} \\
\hline & & \multirow{2}{*}{\multicolumn{3}{|c|}{$\begin{array}{l}4 \mathrm{R} ; 120 \text { to }<350 \\
5 \mathrm{R} ; 350 \text { to }<500\end{array}$}} & \multicolumn{4}{|c|}{$1.1524 \times \ln (\mathrm{uPCR})-1.4229$} & \multicolumn{4}{|c|}{$1.1401 \times \ln (\mathrm{uPCR})-1.2878$} & \multirow{2}{*}{\multicolumn{3}{|c|}{$\begin{array}{l}1.0335 \times \ln (\mathrm{uPCR})-0.9770 \\
1.8136 \times \ln (\mathrm{uPCR})-5.5472\end{array}$}} \\
\hline & & & & & $1.0269 \times$ & $\ln (\mathrm{uPCF}$ & R)-0.6 & & 0.9986 & $\times \ln (\mathrm{uPCR}$ & -0.4592 & & & & \\
\hline & $\mathrm{CE}$ & SE & $t$ & $p>t$ & $95 \% \mathrm{CI}$ & $\mathrm{CE}$ & SE & $t$ & $p>t$ & $95 \% \mathrm{CI}$ & $\mathrm{CE}$ & SE & $t$ & $p>t$ & $95 \% \mathrm{CI}$ \\
\hline \multicolumn{16}{|l|}{$\begin{array}{l}\text { Change in } \\
\text { slope }\end{array}$} \\
\hline $1 \mathrm{R}$ vs $2 \mathrm{R}$ & 0.25 & 0.52 & 0.48 & 0.64 & $\begin{array}{l}-0.80 \\
\text { to } 1.30\end{array}$ & 0.40 & 0.52 & 0.77 & 0.45 & $\begin{array}{c}-0.65 \text { to } \\
1.45\end{array}$ & 0.61 & 0.86 & 0.70 & 0.49 & $\begin{array}{r}-1.14 \\
\quad \text { to } 2.36\end{array}$ \\
\hline $2 \mathrm{R}$ vs $3 \mathrm{R}$ & 0.93 & 0.38 & 2.43 & 0.02 & $\begin{array}{r}0.16- \\
1.69\end{array}$ & 0.98 & 0.38 & 2.58 & 0.01 & $0.21-1.74$ & 0.37 & 0.63 & 0.58 & 0.56 & $\begin{array}{l}-0.91 \\
\text { to } 1.65\end{array}$ \\
\hline $3 R$ vs $4 R$ & -0.40 & 0.29 & -1.38 & 0.17 & $\begin{array}{l}-0.97 \\
\text { to } 0.18\end{array}$ & -0.52 & 0.29 & -1.81 & 0.08 & $\begin{array}{c}-1.09 \text { to } \\
0.06\end{array}$ & -0.16 & 0.50 & -0.33 & 0.75 & $\begin{array}{l}-1.17 \\
\text { to } 0.85\end{array}$ \\
\hline $4 R$ vs $5 R$ & -0.13 & 0.18 & -0.69 & 0.50 & $\begin{array}{l}-0.49 \\
\text { to } 0.24\end{array}$ & -0.14 & 0.18 & -0.77 & 0.44 & $\begin{array}{c}-0.51 \text { to } \\
0.23\end{array}$ & 0.78 & 0.54 & 1.45 & 0.16 & $\begin{array}{l}-0.32 \\
\text { to } 1.88\end{array}$ \\
\hline
\end{tabular}

$u A C R$ urinary albumin-to-creatinine ratio; $u P C R$ urinary protein-to-creatinine ratio, ln natural logarithm; $C E$ coefficient; $S E$ standard error; $C I$ confidence interval, Unit of uPCR, $\mathrm{mg} / \mathrm{gCr}$; Unit of $\mathrm{uACR}, \mathrm{mg} / \mathrm{gCr}$ 


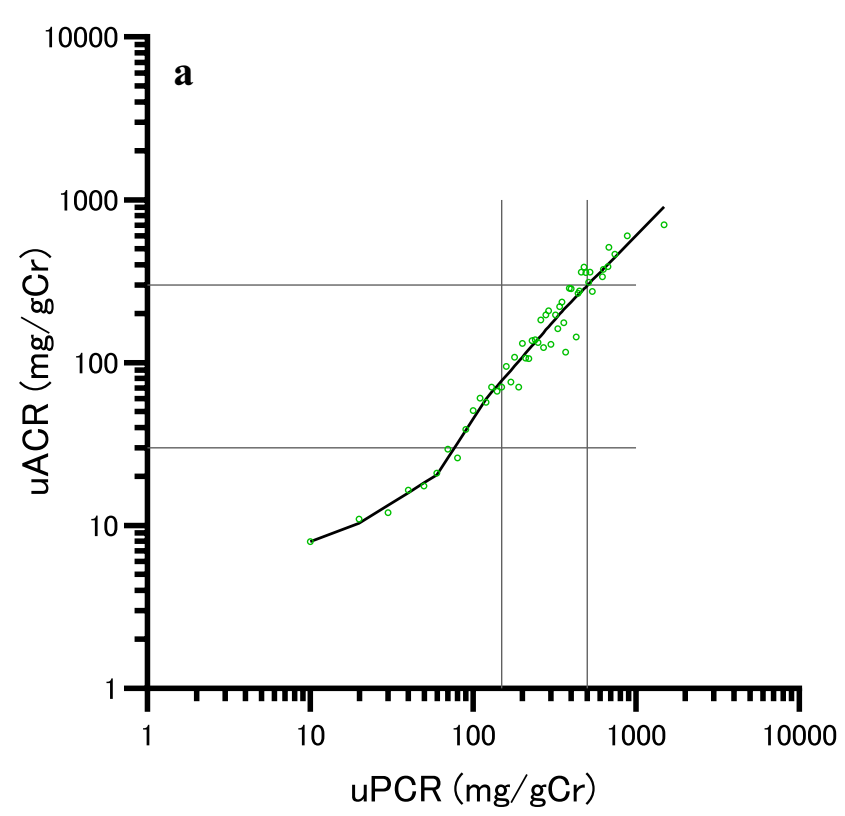

Fig. 1 The associations between uPCR and median uACR by a linear spline using 4 knots. a non-diabetic and diabetic patients. Adjusted R-squared 0.965, AIC - 103.7. O: Median uACR of non-diabetic and diabetic patients. b The red line, non-diabetics (Adjusted R-squared 0.967, AIC - 100.2); blue line, diabetics (Adjusted R-squared 0.899, AIC - 36.7). $\square$ : Median uACR of non-diabetic patients. $\triangle$ : Median

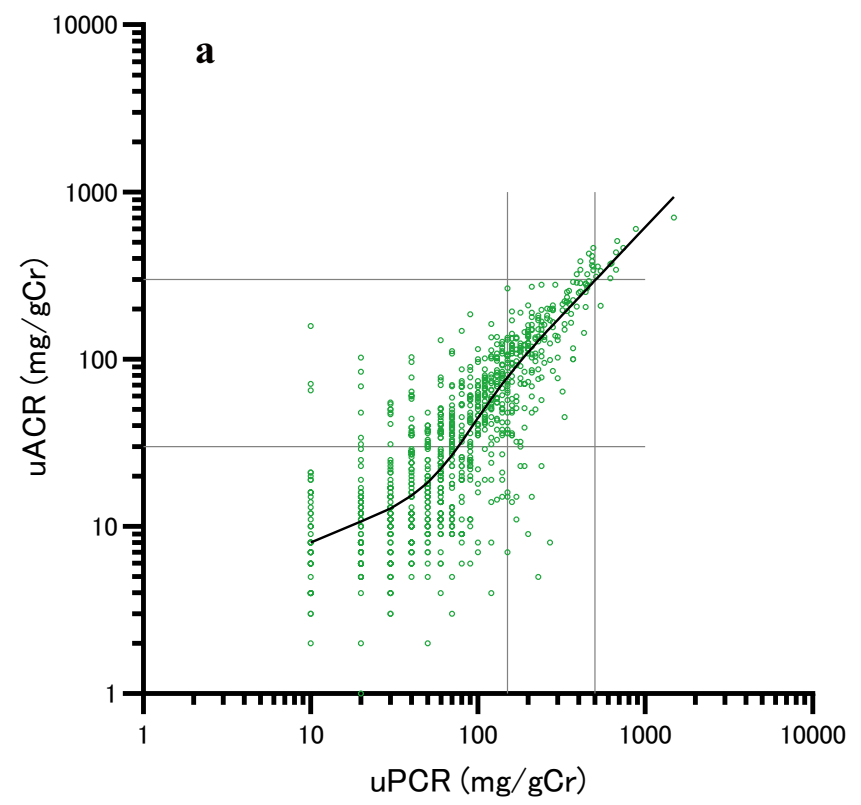

Fig. 2 The associations between uPCR and median uACR by a restricted cubic spline using 4 knots. a non-diabetic and diabetic patients. Adjusted R-squared 0.966, AIC - 107.2. O: uACR of nondiabetic and diabetic patients. b The red line, non-diabetics (Adjusted R-squared 0.967, AIC - 101.3); blue line, diabetics (Adjusted

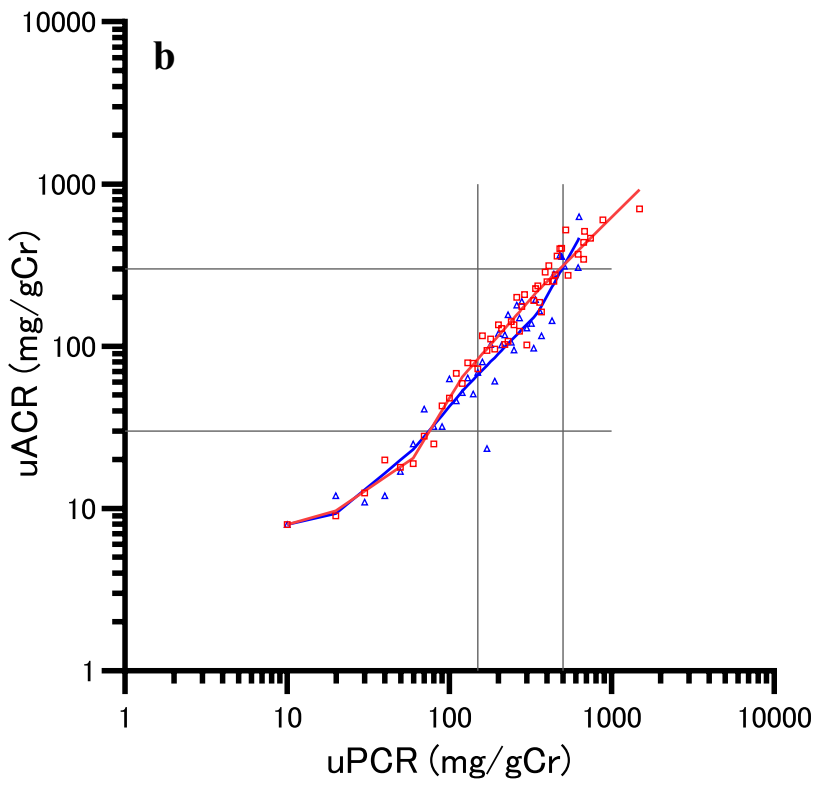

uACR of diabetic patients. The 4 knots were at percentiles $5,35,65$, 95 of 846 measurements, corresponding to uPCR of 20,60,120, and $350 \mathrm{mg} / \mathrm{gCr}$. The two vertical lines indicate uPCR 150 and $500 \mathrm{mg} /$ $\mathrm{gCr}$, respectively, while the two horizontal lines indicate uACR 30 and $300 \mathrm{mg} / \mathrm{gCr}$, respectively

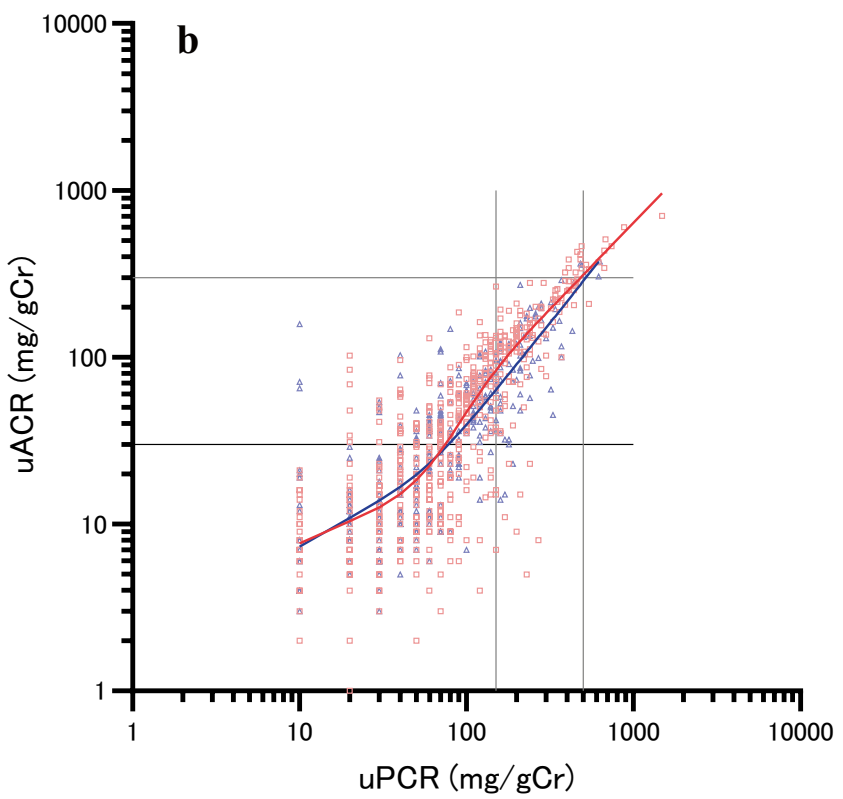

R-squared 0.899, AIC -38.4). $\square$ : uACR of non-diabetic patients. $\triangle$ : uACR of diabetic patients. The two vertical lines indicate uPCR 150 and $500 \mathrm{mg} / \mathrm{gCr}$, respectively, while the two horizontal lines indicate $\mathrm{uACR} 30$ and $300 \mathrm{mg} / \mathrm{gCr}$, respectively 
Table 5 The analysis to discriminate uACR below and above each value in nondiabetic patients by the receiver operating characteristics curve $(n=591)$
Table 6 The analysis to discriminate $\mathrm{uACR}$ below and above each value in diabetic patients by the receiver operating characteristics curve $(n=318)$

\begin{tabular}{|c|c|c|c|c|c|c|c|}
\hline $\begin{array}{l}\text { uACR (X } \\
\text { mg/gCr) }\end{array}$ & $n(\geq \mathrm{X} \mathrm{mg/gCr})$ & AUC & AUC 95\% CI & $p$ & $\begin{array}{l}\mathrm{uPCR}(\mathrm{g} / \\
\mathrm{Cr}) \mathrm{CO}(\mathrm{YI})\end{array}$ & Sensitivity & specificity \\
\hline 10 & 493 & 0.827 & $0.789-0.865$ & $<0.0001$ & 0.06 & 0.72 & 0.82 \\
\hline 20 & 370 & 0.852 & $0.821-0.883$ & $<0.0001$ & 0.09 & 0.67 & 0.92 \\
\hline 30 & 314 & 0.878 & $0.850-0.906$ & $<0.0001$ & 0.09 & 0.76 & 0.89 \\
\hline 40 & 264 & 0.907 & $0.882-0.932$ & $<0.0001$ & 0.09 & 0.85 & 0.87 \\
\hline 50 & 233 & 0.922 & $0.898-0.945$ & $<0.0001$ & 0.10 & 0.85 & 0.88 \\
\hline 60 & 202 & 0.931 & $0.909-0.954$ & $<0.0001$ & 0.11 & 0.87 & 0.89 \\
\hline 70 & 176 & 0.948 & $0.929-0.967$ & $<0.0001$ & 0.11 & 0.92 & 0.87 \\
\hline 80 & 153 & 0.950 & $0.931-0.970$ & $<0.0001$ & 0.12 & 0.92 & 0.86 \\
\hline 90 & 142 & 0.956 & $0.938-0.974$ & $<0.0001$ & 0.13 & 0.92 & 0.88 \\
\hline 100 & 132 & 0.956 & $0.937-0.974$ & $<0.0001$ & 0.13 & 0.92 & 0.87 \\
\hline 110 & 113 & 0.959 & $0.944-0.975$ & $<0.0001$ & 0.14 & 0.93 & 0.86 \\
\hline 120 & 98 & 0.961 & $0.945-0.978$ & $<0.0001$ & 0.15 & 0.92 & 0.87 \\
\hline 130 & 87 & 0.962 & $0.944-0.980$ & $<0.0001$ & 0.15 & 0.94 & 0.86 \\
\hline 140 & 71 & 0.973 & $0.958-0.988$ & $<0.0001$ & 0.16 & 0.96 & 0.87 \\
\hline 150 & 65 & 0.975 & $0.960-0.991$ & $<0.0001$ & 0.20 & 0.91 & 0.93 \\
\hline 300 & 18 & 0.996 & $0.993-1.000$ & $<0.0001$ & 0.39 & 1.00 & 0.98 \\
\hline
\end{tabular}

$u A C R$ urinary albumin-to-creatinine ratio; $u P C R$ urinary protein-to-creatinine ratio; $A U C$ area under the curve $\mathrm{CO}(\mathrm{YI})$, cut-off value using Youden's index; $C I$ confidence interval

\begin{tabular}{llllllll}
\hline $\begin{array}{l}\text { uACR }(X \\
\mathrm{mg} / \mathrm{gCr})\end{array}$ & $\mathrm{n}(\geq \mathrm{X} \mathrm{mg} / \mathrm{gCr})$ & AUC & AUC 95\% CI & $p$ & $\begin{array}{l}\text { uPCR (g/ } \\
\text { Sr) CO(YI) }\end{array}$ & Sensitivity & specificity \\
\hline 10 & 276 & 0.820 & $0.768-0.872$ & $p<0.0001$ & 0.06 & 0.66 & 0.88 \\
20 & 208 & 0.862 & $0.821-0.903$ & $p<0.0001$ & 0.06 & 0.81 & 0.84 \\
30 & 169 & 0.889 & $0.851-0.926$ & $p<0.0001$ & 0.07 & 0.83 & 0.83 \\
40 & 137 & 0.887 & $0.848-0.927$ & $p<0.0001$ & 0.10 & 0.76 & 0.90 \\
50 & 110 & 0.892 & $0.851-0.934$ & $p<0.0001$ & 0.10 & 0.84 & 0.86 \\
60 & 93 & 0.888 & $0.843-0.933$ & $p<0.0001$ & 0.10 & 0.85 & 0.81 \\
70 & 80 & 0.889 & $0.842-0.937$ & $p<0.0001$ & 0.12 & 0.83 & 0.83 \\
80 & 65 & 0.909 & $0.861-0.957$ & $p<0.0001$ & 0.13 & 0.86 & 0.85 \\
90 & 56 & 0.905 & $0.851-0.960$ & $p<0.0001$ & 0.15 & 0.84 & 0.89 \\
100 & 50 & 0.895 & $0.834-0.955$ & $p<0.0001$ & 0.16 & 0.80 & 0.89 \\
110 & 40 & 0.912 & $0.845-0.979$ & $p<0.0001$ & 0.16 & 0.90 & 0.88 \\
120 & 36 & 0.914 & $0.842-0.986$ & $p<0.0001$ & 0.16 & 0.92 & 0.87 \\
130 & 32 & 0.914 & $0.833-0.994$ & $p<0.0001$ & 0.20 & 0.91 & 0.94 \\
140 & 28 & 0.901 & $0.810-0.993$ & $p<0.0001$ & 0.20 & 0.89 & 0.93 \\
150 & 23 & 0.942 & $0.867-1.018$ & $p<0.0001$ & 0.21 & 0.96 & 0.93 \\
300 & 5 & 1.000 & $1.000-1.000$ & 0.00012 & 0.48 & 1.00 & 1.00 \\
\hline
\end{tabular}

$u A C R$ urinary albumin-to-creatinine ratio; $u P C R$ urinary protein-to-creatinine ratio; $A U C$ area under the curve $\mathrm{CO}(\mathrm{YI})$, cut-off value using Youden's index; $C I$ confidence interval correlation between changes in the $\mathrm{UACR}$ and $\mathrm{UPCR}$ was examined by a simple regression analysis.

The subjects for the examination on the intra- and interday variance of uPCR and uACR of the same sample include patients who were regularly visiting a hospital with lifestyle-related disease in December 2020, had a uPCR of less than $0.5 \mathrm{~g} / \mathrm{gCr}$ at their last outpatient visit within 3 months prior, and used the morning outpatient service. In the morning of day 0 , serum creatinine was measured and a urinalysis and sediment testing of the 
morning urine was carried out. The first measurement was performed in the morning of day 0 and urinary protein, urinary albumin, and urinary creatinine were measured at the same time. The specimens were then stored in the refrigerator at 5 to 7 degrees. The second

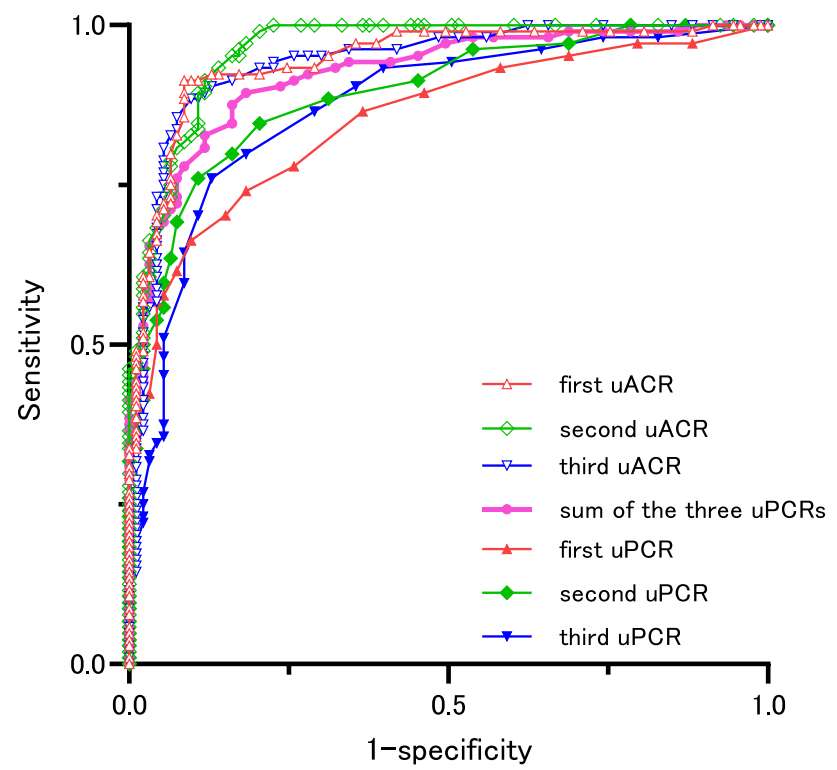

Fig. 3 Differentiation of patients with normoalbuminuria and microand macroalbuminuria by the the first $\mathrm{uACR}(\triangle)$, second uACR $(\diamond)$, third uACR $(\nabla)$, first uPCR $(\boldsymbol{\Delta})$, second uPCR $(\boldsymbol{\nabla})$, third uPCR $(\boldsymbol{\nabla})$, and sum of the three uPCR values $(\mathbf{O})$, according to an ROC analysis measurement was carried out $3 \mathrm{~h}$ after but within $4 \mathrm{~h}$ of the first measurement on the same day, with the urinary protein, albumin, and creatinine measured at the same time after leaving the specimens for $15 \mathrm{~min}$ at room temperature. Thereafter, we plugged the specimens, wrapped them with parafilm, and cryopreserved them at -80 degrees. The third and fourth measurements were carried out on day 3 or 4 . The specimens were thawed with running water at 11:30 AM and left at room temperature from 11:40 AM, after which the urinary protein, albumin, and creatinine were measured at 12:00 PM, twice consecutively within $3 \mathrm{~min}$. Patients with $\mathrm{uPCR} \geq 0.5 \mathrm{~g} / \mathrm{gCr}$ in the morning of day 0 were not excluded.

For urinary protein, urinary albumin, urinary creatinine, uPCR, and uACR, we calculated the mean of the two measurements used for comparison. For urinary protein, urinary albumin, and urinary creatinine, the mean values were classified into three groups in ascending order. For uPCR and UACR, the mean values of UPCR were divided into two groups: less than $0.15 \mathrm{~g} / \mathrm{gCr}$; and $0.15 \mathrm{~g} / \mathrm{gCr}$ or more. We then calculated the intraday correlation and interday correlation within each group, the median, and the IQR of the bias of the later measurements against the previous measurements. In each of the four measurements, we calculated the $\mathrm{CO}$ of uPCR and urinary protein concentration estimating a uACR of $30 \mathrm{mg} / \mathrm{gCr}$ or more, which were measured simultaneously. The $\mathrm{CO}$ of uPCR estimating the mean of the four uACR values $\geq 30 \mathrm{mg} / \mathrm{gCr}$ was also calculated and used for comparison.

Table 7 Cut-off value (YI) of the uPCR and uACR distinguishing patients with normoalbuminuria $(n=93)$ and micro- and macroalbuminuria $(n=104)$ in non-diabetic patients

\begin{tabular}{lccclllllll}
\hline Test variables & $1 \mathrm{uPCR}$ & $2 \mathrm{uPCR}$ & $3 \mathrm{uPCR}$ & $1.2 \mathrm{uPCR}$ & $2.3 \mathrm{uPCR}$ & $1.3 \mathrm{uPCR}$ & $1.2 .3 \mathrm{uPCR}$ & $1 \mathrm{uACR}$ & $2 \mathrm{uACR}$ & $3 \mathrm{uACR}$ \\
\hline ROC curve area & 0.854 & 0.894 & 0.873 & $0.912^{\mathrm{a}}$ & $0.911^{\mathrm{b}}$ & $0.904^{\mathrm{c}}$ & $0.924^{\mathrm{d}}$ & $0.945^{\mathrm{e}}$ & $0.961^{\mathrm{f}}$ & $0.941^{\mathrm{g}}$ \\
Standard error & 0.027 & 0.022 & 0.026 & 0.020 & 0.021 & 0.021 & 0.018 & 0.01598 & 0.012 & 0.017 \\
$p$ value & $<0.0001$ & $<0.0001$ & $<0.0001$ & $<0.0001$ & $<0.0001$ & $<0.0001$ & $<0.0001$ & $<0.0001$ & $<0.0001$ & $<0.0001$ \\
CO $(\text { YI })^{\mathrm{h}}$ & 0.10 & 0.09 & 0.09 & 0.16 & 0.16 & 0.17 & 0.23 & 31 & 31 & 30 \\
Sensitivity & 0.66 & 0.76 & 0.76 & 0.85 & 0.85 & 0.84 & 0.88 & 0.91 & 0.91 & 0.89 \\
Specificity & 0.82 & 0.89 & 0.87 & 0.87 & 0.86 & 0.85 & 0.84 & 0.91 & 0.88 & 0.90 \\
\hline
\end{tabular}

1, 2, 3 uPCR denote first, second, and third uPCR, respectively. 1.2uPCR, 2.3uPCR, 1,3uPCR each denote the sum of the two indicated uPCRs. 1.2.3uPCR denotes the sum of the three uPCRs. 1, 2, 3uACR denotes first, second, and third uACR, respectively

uACR, urinary albumin-to-creatinine ratio; uPCR, urinary protein-to-creatinine ratio, $\mathrm{CO}(\mathrm{YI})$, cut-off value using Youden's index by the receiver operating characteristics curve

${ }^{\mathrm{a}} \mathrm{vs.} 1 \mathrm{uPCR}, p=0.0005$

bs. 3uPCR, $p=0.005$

${ }^{\mathrm{c}} \mathrm{vs} 3 \mathrm{uPCR}, p=0.047$

dvs. 1uPCR, $p=0.0005$; vs. 2 uPCR, $p=0.047$; vs. 3 uPCR, $p=0.003$

evs. 1uPCR, $p=0.0002$; vs. 2 uPCR, $p=0.045$; vs. 3 uPCR, $p=0.020$

f vs. 1 uPCR, $p=0.0002$; vs. 2 uPCR, $p=0.001$; vs. 3 uPCR, $p=0.001$; vs. $1.2 \mathrm{UP}, p=0.018$, vs. $2.3 \mathrm{UP}, p=0.015$; vs. $1.3 \mathrm{UP}, p=0.013$

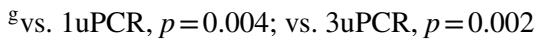

${ }^{\mathrm{h}}$ Unit of uPCR, g/gCr; Unit of uACR, $\mathrm{mg} / \mathrm{gCr}$ 
Table 8 Cut-off value (YI) of uPCR discriminating uACR $\geq 30 \mathrm{mg} / \mathrm{gCr}$ in patients with or without diabetes mellitus according to the age, sex, GFR category $(n=591)$

\begin{tabular}{|c|c|c|c|c|c|c|c|}
\hline \multirow{3}{*}{$\begin{array}{l}\text { Cause } \\
\text { Test variables }\end{array}$} & \multicolumn{7}{|c|}{ Non-diabetes mellitus } \\
\hline & \multirow[b]{2}{*}{$<65$} & \multirow{2}{*}{$\begin{array}{l}\text { Age (years) } \\
65 \text { to }<80\end{array}$} & \multirow[b]{2}{*}{$\geq 80$} & \multirow{2}{*}{$\begin{array}{l}\text { Sex } \\
\text { Male }\end{array}$} & \multirow[b]{2}{*}{ Female } & \multicolumn{2}{|c|}{ GFR category } \\
\hline & & & & & & G1-3a & G3b-4 \\
\hline$n$ & 201 & 228 & 162 & 372 & 219 & 375 & 216 \\
\hline$n(\mathrm{uACR} \geq 30 \mathrm{mg} / \mathrm{gCr})$ & 108 & 108 & 98 & 187 & 127 & 185 & 129 \\
\hline ROC curve area & 0.896 & 0.862 & 0.863 & 0.861 & 0.908 & 0.865 & 0.892 \\
\hline Standard error & 0.022 & 0.026 & 0.030 & 0.020 & 0.021 & 0.020 & 0.022 \\
\hline$p$ value & $<0.0001$ & $<0.0001$ & $<0.0001$ & $<0.0001$ & $<0.0001$ & $<0.0001$ & $<0.0001$ \\
\hline $\mathrm{CO}(\mathrm{YI})(\mathrm{g} / \mathrm{gCr})$ & 0.09 & 0.09 & 0.08 & 0.09 & 0.09 & 0.09 & 0.10 \\
\hline Sensitivity & 0.74 & 0.72 & 0.84 & 0.74 & 0.78 & 0.72 & 0.79 \\
\hline Specificity & 0.91 & 0.92 & 0.80 & 0.87 & 0.94 & 0.91 & 0.89 \\
\hline Cause & \multicolumn{7}{|c|}{ Diabetes mellitus } \\
\hline \multirow[t]{2}{*}{ Test variables } & & \multicolumn{2}{|l|}{ Age (years) } & \multicolumn{2}{|l|}{ Sex } & \multicolumn{2}{|c|}{ GFR category } \\
\hline & $<65$ & 65 to $<80$ & $\geq 80$ & Male & Female & G1-3a & G3b-4 \\
\hline$n$ & 123 & 153 & 42 & 210 & 108 & 249 & 69 \\
\hline$n(\mathrm{uACR} \geq 30 \mathrm{mg} / \mathrm{gCr})$ & 56 & 81 & 32 & 105 & 64 & 117 & 52 \\
\hline ROC curve area & 0.879 & 0.889 & 0.878 & 0.909 & 0.842 & 0.872 & 0.912 \\
\hline Standard error & 0.033 & 0.029 & 0.069 & 0.022 & 0.037 & 0.025 & 0.036 \\
\hline$p$ value & $<0.0001$ & $<0.0001$ & 0.0004 & $<0.0001$ & $<0.0001$ & $<0.0001$ & $<0.0001$ \\
\hline $\mathrm{CO}(\mathrm{YI})(\mathrm{g} / \mathrm{gCr})$ & 0.06 & 0.07 & 0.08 & 0.07 & 0.11 & 0.07 & 0.07 \\
\hline Sensitivity & 0.89 & 0.83 & 0.84 & 0.88 & 0.63 & 0.80 & 0.89 \\
\hline Specificity & 0.76 & 0.85 & 0.90 & 0.87 & 1.00 & 0.84 & 0.77 \\
\hline
\end{tabular}

$u A C R$ urinary albumin-to-creatinine ratio; $u P C R$ urinary protein-to-creatinine ratio, $C O(Y I)$ cut-off value using Youden's index; $G F R$ glomerular filtration rate

All statistical analyses were performed using the BellCurve for Excel (Social Survey Research Information Co., Ltd., Tokyo, Japan), SigmaStat Statistics (Systat software, Inc., USA) and Stata MP version 16 (StataCorp LP, College Station, Texas).

\section{Results}

The subjects included 197 patients with non-diabetic lifestyle-related diseases (age, 68.9 \pm 14.8 years) and 106 patients with diabetes (age, $67.1 \pm 11.6$ years). Table 1 shows the primary lifestyle-related diseases, GFR, and albuminuria category.

Table 2 shows the $\mathrm{uACR}$ and uPCR for each GFR category. While proteinuria was less than the measured sensitivity 44 times (7.4\%) among non-diabetics and 18 times $(5.7 \%)$ among diabetics, albuminuria was less than the measured sensitivity 1 time $(0.7 \%)$ among non-diabetics. The uACR values did not differ according to the GFR category, and the
uPCR values in G4 were higher in comparison to those in G1 among patients with non-diabetic lifestyle-related diseases. In diabetics, the uACR and uPCR values in G3b and 4 were higher in comparison to those in G1-3a. When uPCR/ uACR was assessed, with the exception of one case in which uACR was below the level of sensitivity, the uPCR/uACR values were lower in non-diabetics than in diabetics in G1 and higher in G4(Table 2).

\section{Relationship between the UPCR and UACR}

The study on the relationship between the UPCR and the median UACR indicated that the median UACR increased as the UPCR increased in all patients, non-diabetic patients and diabetic patients, with the median uACR exceeding $30 \mathrm{mg} / \mathrm{gCr}$ at $\mathrm{uPCR} 0.09 \mathrm{~g} / \mathrm{gCr}$ in all patients and non-diabetic patients and at uPCR $0.07 \mathrm{~g} / \mathrm{gCr}$ in diabetic patients. The median uACR corresponding to uPCR $0.15 \mathrm{~g} /$ $\mathrm{gCr}$ was $71 \mathrm{mg} / \mathrm{gCr}$ in the overall patients, $72.5 \mathrm{mg} / \mathrm{gCr}$ in 
Table 9 Cut-off value (YI) of 2nd uPCR discriminating normoalbuminuria and micro- and macroalbuminuria in patients with or without diabetes mellitus according to the age, sex, GFR category

\begin{tabular}{|c|c|c|c|c|c|c|c|c|}
\hline \multirow{3}{*}{ Test variables } & \multicolumn{8}{|c|}{ Non-diabetes mellitus } \\
\hline & \multirow[b]{2}{*}{ All } & \multirow[b]{2}{*}{$<65$} & \multicolumn{2}{|l|}{ Age (years) } & \multicolumn{2}{|l|}{ Sex } & \multicolumn{2}{|c|}{ GFR category } \\
\hline & & & 65 to $<80$ & $\geq 80$ & Male & Female & G1-3a & G3b-4 \\
\hline$n$ & 197 & 67 & 76 & 54 & 124 & 73 & 125 & 72 \\
\hline$n(\geq$ microalbuminuria $)$ & 104 & 35 & 36 & 33 & 61 & 43 & 61 & 43 \\
\hline ROC curve area & 0.894 & 0.949 & 0.876 & 0.829 & 0.893 & 0.902 & 0.917 & 0.840 \\
\hline Standard error & 0.022 & 0.025 & 0.039 & 0.058 & 0.028 & 0.035 & 0.023 & 0.047 \\
\hline$P$ value & $<0.0001$ & $<0.0001$ & $<0.0001$ & $<0.0001$ & $<0.0001$ & $<0.0001$ & $<0.0001$ & $<0.0001$ \\
\hline $\mathrm{CO}(\mathrm{YI})(\mathrm{g} / \mathrm{gCr})$ & 0.09 & 0.09 & 0.09 & 0.07 & 0.09 & 0.09 & 0.07 & 0.10 \\
\hline Sensitivity & 0.76 & 0.83 & 0.69 & 0.85 & 0.77 & 0.74 & 0.85 & 0.74 \\
\hline Specificity & 0.89 & 0.97 & 0.90 & 0.71 & 0.87 & 0.93 & 0.88 & 0.83 \\
\hline \multirow{3}{*}{ Test variables } & \multicolumn{8}{|c|}{ Diabetes mellitus } \\
\hline & & & Age (years) & & Sex & & GFR categ & \\
\hline & All & $<65$ & 65 to $<80$ & $\geq 80$ & Male & Female & G1-3a & G3b-4 \\
\hline$n$ & 106 & 41 & 51 & 14 & 70 & 36 & 83 & 23 \\
\hline$n(\geq$ microalbuminuria $)$ & 55 & 19 & 25 & 11 & 33 & 22 & 39 & 16 \\
\hline ROC curve area & 0.831 & 0.835 & 0.812 & 0.879 & 0.845 & 0.807 & 0.786 & 0.951 \\
\hline Standard error & 0.040 & 0.065 & 0.062 & 0.100 & 0.049 & 0.072 & 0.051 & 0.043 \\
\hline$p$ value & $<0.0001$ & 0.0003 & 0.0001 & 0.0516 & $<0.0001$ & 0.0022 & $<0.0001$ & 0.0007 \\
\hline $\mathrm{CO}(\mathrm{YI})(\mathrm{g} / \mathrm{gCr})$ & 0.06 & 0.06 & 0.10 & 0.15 & 0.06 & 0.12 & 0.06 & 0.07 \\
\hline Sensitivity & 0.86 & 0.90 & 0.68 & 0.73 & 0.91 & 0.59 & 0.80 & 1.00 \\
\hline Specificity & 0.69 & 0.73 & 0.88 & 1.00 & 0.68 & 0.93 & 0.68 & 0.71 \\
\hline
\end{tabular}

Microalbuminuria: urinary albumin-to-creatinine ratio $30-299 \mathrm{mg} / \mathrm{gCr}$ at least twice for 3 measurements

uPCR urinary protein-to-creatinine ratio, $C O(Y I)$ cut-off value using Youden's index; GFR glomerular filtration rate

non-diabetic patients, and $69.0 \mathrm{mg} / \mathrm{gCr}$ in diabetic patients (Table 3).

Regarding the number of knots in the linear spline of the uPCR and the median uACR, as well as the restricted cubic spline, we selected 4 knots, since the AIC values of the models using 4 knots were lower than those using 3 or 5 knots in the overall patients as well as in non-diabetic patients and were nearly equal in diabetic patients.

Table 4 shows the equations to estimate the median uACR from the uPCR using 4-knots linear spline in the overall patients, in non-diabetic patients, and diabetic patients. Regarding the linear spline, the uPCR corresponding to the median uACR $30 \mathrm{mg} / \mathrm{gCr}$ was $0.077 \mathrm{~g} / \mathrm{gCr}$ in the overall patients, while the median uACR corresponding to uPCR $0.15 \mathrm{~g} / \mathrm{gCr}$ was $78 \mathrm{mg} / \mathrm{gCr}$ (Fig. 1a). In non-diabetic and diabetic patients, the uPCR corresponding to the median uACR $30 \mathrm{mg} / \mathrm{gCr}$ was 0.075 and $0.075 \mathrm{~g} / \mathrm{gCr}$, respectively, while the median ACR corresponding to uPCR0.15 g/gCr was 83 and $67 \mathrm{mg} / \mathrm{gCr}$ (Fig. 1b), respectively. The median uACR corresponding to a uPCR of $0.50 \mathrm{~g} / \mathrm{gCr}$ in the overall patients, non-diabetic patients, and diabetic patients was
297, 313, and $306 \mathrm{mg} / \mathrm{gCr}$, respectively. When examining the shift of inclination in $\Delta \ln ($ median $\mathrm{uACR}) / \Delta \ln (\mathrm{uPCR})$ before and after each knot, it increased significantly at PCR $0.060 \mathrm{~g} / \mathrm{gCr}$ overall, with a significant increase in non-diabetics when looking at it by disease. Although it did increase in diabetics as well, it was not significant.

The relationship between the median uACR and uPCR was the same for the restricted cubic spline (Fig. 2 a,b).

The relationship between the $\mathrm{uACR}$ and the $\mathrm{CO}$ of the uPCR for determining whether it was greater or less than the uACR (Tables 5, 6).

The uPCR that differentiated the uACR (for uACR $10-300 \mathrm{mg} / \mathrm{gCr}$ ) was examined using an ROC curve for a total of 591 measurements in patients with non-diabetic lifestyle-related diseases and a total of 318 measurements in patients with diabetes. For uACR $10-300 \mathrm{mg} / \mathrm{gCr}$, the higher the uACR value, the higher the AUC of the ROC curve, the CO of uPCR, the sensitivity, and the specificity. A uACR of greater or less than $30 \mathrm{mg} / \mathrm{gCr}$ was differentiated with a uPCR of $0.09 \mathrm{~g} / \mathrm{gCr}$ in patients with non-diabetic lifestyle diseases (AUC 0.88, Sn 0.76, Sp 0.89), and a uPCR 
Table 10 Sensitivity, specificity and Youden,s index for discriminating non-diabetic patients with $\mathrm{uACR} \geq 30 \mathrm{mg} / \mathrm{gCr}$ or microand macroalbuminuria by uPCR according to GFR category

\begin{tabular}{|c|c|c|c|c|c|}
\hline \multirow[t]{2}{*}{ GFR category } & \multirow[t]{2}{*}{ Albuminuria category } & & \multicolumn{3}{|c|}{$\mathrm{uPCR}(\mathrm{g} / \mathrm{gCr})$} \\
\hline & & & $\geq 0.07$ & $\geq 0.09$ & $\geq 0.15$ \\
\hline \multirow[t]{3}{*}{ G1-4 } & \multirow[t]{3}{*}{$\mathrm{uACR} \geq 30 \mathrm{mg} / \mathrm{gCr}$} & Sensitivity & 0.84 & 0.76 & 0.47 \\
\hline & & Specificity & 0.77 & 0.89 & 0.98 \\
\hline & & Youden's Index & 0.61 & 0.65 & 0.44 \\
\hline \multirow[t]{3}{*}{ G1-4 } & \multirow[t]{3}{*}{$\geq$ Microalbuminuria } & Sensitivity & 0.85 & 0.76 & 0.50 \\
\hline & & Specificity & 0.80 & 0.89 & 0.98 \\
\hline & & Youden's Index & 0.64 & 0.65 & 0.48 \\
\hline \multirow[t]{3}{*}{ G1-3a } & \multirow[t]{3}{*}{$\mathrm{uACR} \geq 30 \mathrm{mg} / \mathrm{gCr}$} & Sensitivity & 0.82 & 0.72 & 0.46 \\
\hline & & Specificity & 0.81 & 0.91 & 0.97 \\
\hline & & Youden's Index & 0.62 & 0.64 & 0.43 \\
\hline \multirow[t]{3}{*}{ G1-3a } & \multirow[t]{3}{*}{$\geq$ Microalbuminuria } & Sensitivity & 0.85 & 0.75 & 0.54 \\
\hline & & Specificity & 0.88 & 0.95 & 1.00 \\
\hline & & Youden's Index & 0.73 & 0.71 & 0.54 \\
\hline \multirow[t]{3}{*}{ G3b-4 } & \multirow[t]{3}{*}{$\mathrm{uACR} \geq 30 \mathrm{mg} / \mathrm{gCr}$} & Sensitivity & 0.88 & 0.80 & 0.47 \\
\hline & & Specificity & 0.68 & 0.85 & 0.98 \\
\hline & & Youden's Index & 0.55 & 0.65 & 0.45 \\
\hline \multirow[t]{3}{*}{ G3b-4 } & \multirow[t]{3}{*}{$\geq$ Microalbuminuria } & Sensitivity & 0.84 & 0.77 & 0.44 \\
\hline & & Specificity & 0.62 & 0.76 & 0.93 \\
\hline & & Youden's Index & 0.46 & 0.53 & 0.37 \\
\hline
\end{tabular}

Microalbuminuria: urinary albumin-to-creatinine ratio $30-299 \mathrm{mg} / \mathrm{gCr}$ at least twice for 3 measurements $u A C R$ urinary albumin-to-creatinine ratio; $u P C R$ urinary protein-to-creatinine ratio; GFR glomerular filtration rate

\begin{tabular}{|c|c|c|c|c|c|c|c|}
\hline \multirow[b]{2}{*}{ GFR category } & \multirow[b]{2}{*}{$\mathrm{uACR}(\mathrm{mg} / \mathrm{gCr})$} & \multicolumn{6}{|c|}{ uPCR (g/gCr) } \\
\hline & & $<0.07$ & 0.07 to $<0.15$ & $<0.09$ & 0.09 to $<0.15$ & 0.15 to $<0.50$ & $\geq 0.50$ \\
\hline \multirow[t]{4}{*}{ G1-3a } & $0-29$ & 153 & 32 & 173 & 12 & 5 & 0 \\
\hline & $0-9$ & 59 & 9 & 64 & 4 & 0 & 0 \\
\hline & $10-19$ & 70 & 11 & 78 & 3 & 4 & 0 \\
\hline & $20-29$ & 24 & 12 & 31 & 5 & 1 & 0 \\
\hline \multirow[t]{6}{*}{ G1-3a } & $30-299$ & 34 & 66 & 51 & 49 & 75 & 1 \\
\hline & $30-59$ & 28 & 32 & 40 & 20 & 7 & 0 \\
\hline & $60-99$ & 6 & 25 & 11 & 20 & 12 & 0 \\
\hline & $100-149$ & 0 & 8 & 0 & 8 & 28 & 0 \\
\hline & $150-199$ & 0 & 1 & 0 & 1 & 12 & 0 \\
\hline & 200-299 & 0 & 0 & 0 & 0 & 16 & 1 \\
\hline G1-3a & $\geq 300$ & 0 & 0 & 0 & 0 & 6 & 3 \\
\hline \multirow[t]{4}{*}{ G3b-4 } & $0-29$ & 59 & 26 & 74 & 11 & 2 & 0 \\
\hline & $0-9$ & 26 & 3 & 29 & 0 & 1 & 0 \\
\hline & $10-19$ & 23 & 15 & 32 & 6 & 0 & 0 \\
\hline & $20-29$ & 10 & 8 & 13 & 5 & 1 & 0 \\
\hline \multirow[t]{6}{*}{ G3b-4 } & 30-299 & 16 & 52 & 26 & 42 & 52 & 0 \\
\hline & $30-59$ & 12 & 28 & 21 & 19 & 5 & 0 \\
\hline & 60-99 & 2 & 16 & 3 & 15 & 9 & 0 \\
\hline & $100-149$ & 2 & 7 & 2 & 7 & 22 & 0 \\
\hline & 150-199 & 0 & 1 & 0 & 1 & 7 & 0 \\
\hline & 200-299 & 0 & 0 & 0 & 0 & 9 & 0 \\
\hline G3b-4 & $\geq 300$ & 0 & 0 & 0 & 0 & 3 & 6 \\
\hline
\end{tabular}

GFR glomerular filtration rate; $u A C R$ urinary albumin-to-creatinine ratio; $u P C R$ urinary protein-to-creatinine ratio 


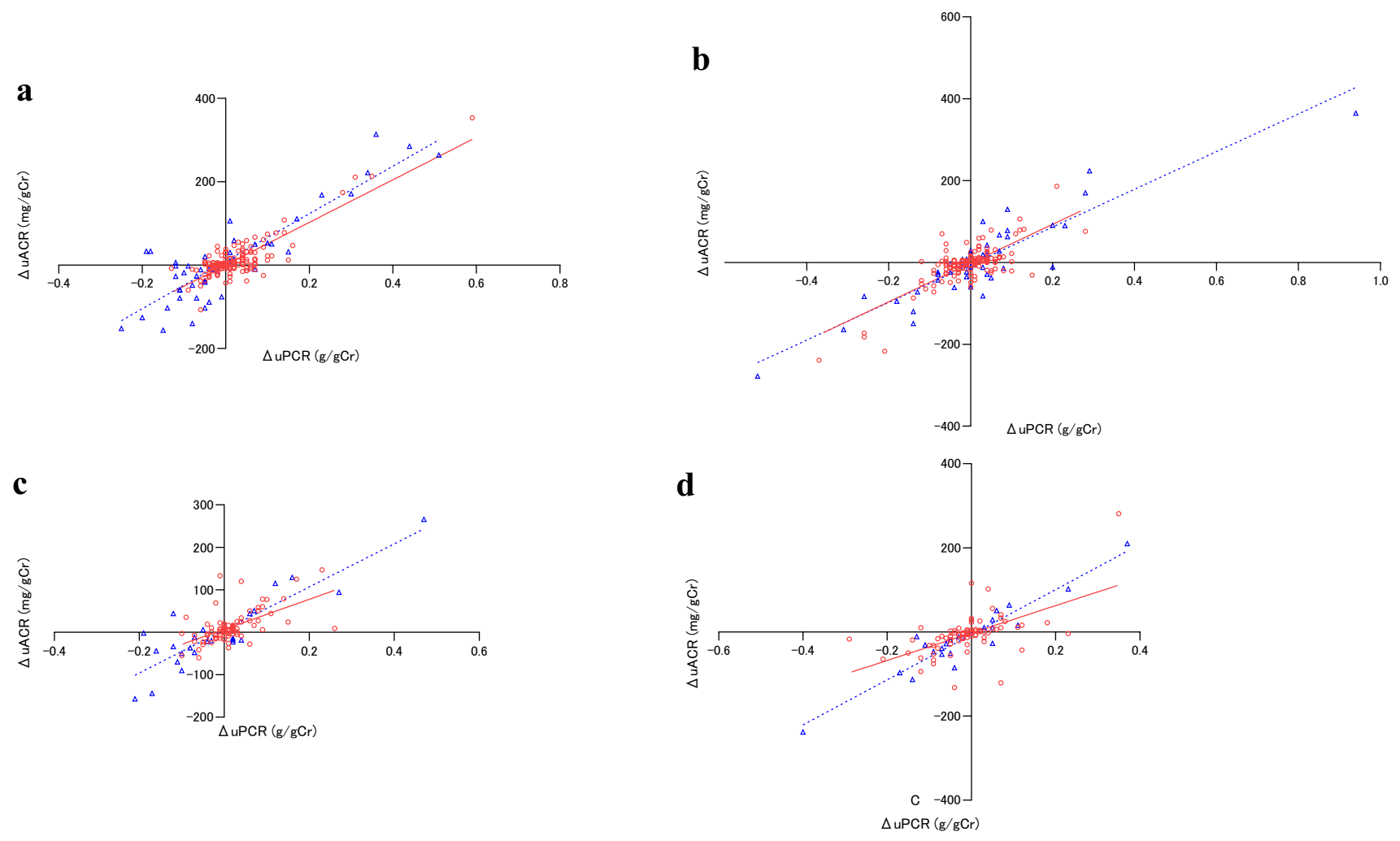

Fig. 4 (a) Association of differences between the second and first uPCR value and the uACR in non-diabetic patients. $\bigcirc$ : first $\mathrm{uPCR}<0.15 \mathrm{~g} / \mathrm{gCr}, \quad-$ : $\quad \Delta \mathrm{uACR}=510.4 \times \Delta \mathrm{uPCR}+0.4$, $R=0.85, \quad p=6.5 \times 10^{-44} . \quad \triangle$ : first $\mathrm{uPCR} \geq 0.15 \mathrm{~g} / \mathrm{gCr}, \quad---$ : $\Delta \mathrm{uACR}=569.9 \times \Delta \mathrm{uPCR}+9.2, \quad R=0.88, \quad p=3.1 \times 10^{-16} . \quad$ b Association of differences between the third and second uPCR value and the uACR in non-diabetic patients. $\bigcirc$ : first $\mathrm{uPCR}<0.15 \mathrm{~g} /$ $\mathrm{gCr}, \quad-$ : $\quad \Delta \mathrm{uACR}=473.1 \times \Delta \mathrm{uPCR}-1.5, \quad R=0.76, \quad p=3.8 \times 10^{-29}$. $\triangle$ : first $\quad$ uPCR $\geq 0.15 \mathrm{~g} / \mathrm{gCr}, \quad---: \quad \Delta \mathrm{uACR}=460.6 \times \Delta \mathrm{uPCR}-5.4$, $\mathrm{R}=0.90, \mathrm{p}=4.1 \times 10^{-18}$. c Association of differences between the

of $0.07 \mathrm{~g} / \mathrm{gCr}$ in patients with diabetes (AUC 0.89, Sn 0.83, Sp 0.83). The CO of uPCR $0.15 \mathrm{~g} / \mathrm{gCr}$, according to the Youden's index, differentiated a uACR of greater or less than $120 \mathrm{mg} / \mathrm{gCr}$ in patients with non-diabetic lifestyle diseases (AUC 0.96, Sn 0.92, Sp 0.87), and uACR greater or less than $90 \mathrm{mg} / \mathrm{gCr}$ for diabetes (AUC 0.91, Sn 0.84, Sp 0.89).

Differentiation of microalbuminuria by the UPCR and uACR in non-diabetic patients (Fig. 3, Table 7).

A single uPCR was useful for differentiating normoalbuminuria and micro- and macroalbuminuria in both nondiabetics and diabetics. The $\mathrm{CO}$ of the second uPCR measurement was $0.09 \mathrm{~g} / \mathrm{gCr}$ in non-diabetics (AUC 0.89, $\mathrm{Sn}$ $0.76, \mathrm{Sp} 0.89$ ) and $0.06 \mathrm{~g} / \mathrm{gCr}$ in diabetics (AUC 0.83, Sn $0.86, \mathrm{Sp} 0.69$ ). The sum of the uPCRs measured twice, at the first and second, as well as the second and third measurements, was useful for the differentiation of micro- and macroalbuminuria in non-diabetics (AUC 0.91, CO $0.16 \mathrm{~g} /$ second and first uPCR value and the uACR in diabetic patients. $O$ : first $\mathrm{uPCR}<0.15 \mathrm{~g} / \mathrm{gCr},-$ : $\Delta \mathrm{uACR}=353.9 \times \Delta \mathrm{uPCR}+6.8$, $R=0.59, \quad p=8.1 \times 10^{-9} . \quad \Delta: \quad$ first $\quad \mathrm{uPCR} \geq 0.15 \quad \mathrm{~g} / \mathrm{gCr}, \quad---$ $\Delta \mathrm{uACR}=507.1 \times \Delta \mathrm{uPCR}+5.3, \quad \mathrm{R}=0.88, \quad \mathrm{p} 8.7=\times 10^{-9} . \quad$ d Association of differences between the third and second uPCR value and the uACR in diabetic patients. $\bigcirc$ : first $\mathrm{uPCR}<0.15 \mathrm{~g} / \mathrm{gCr},-$ : $\Delta \mathrm{uACR}=326.1 \times \Delta \mathrm{uPCR}-2.6, \quad R=0.57, \quad p=2.7 \times 10^{-8} . \quad \triangle$ : first $\mathrm{uPCR} \geq 0.15 \mathrm{~g} / \mathrm{gCr}, \quad---: \quad \Delta \mathrm{uACR}=536.4 \times \Delta \mathrm{uPCR}-6.5, \quad R=0.94$, $p=1.1 \times 10^{-12}$

$\mathrm{gCr})$. The sum of the three uPCRs was useful for differentiating microalbuminuria (AUC 0.92, CO uPCR $0.23 \mathrm{~g} /$ $\mathrm{gCr}, \mathrm{Sn} 0.88, \mathrm{Sp} \mathrm{0.84)}$ and was significantly more useful than the single uPCR. The second uACR was useful for differentiating microalbuminuria (AUC 0.96, CO uACR $31 \mathrm{mg} / \mathrm{gCr}$, Sn 0.91 , Sp 0.88) and significantly more useful than a single uPCR or the sum of two uPCRs; it also tended to be useful for comparing the sum of three uPCRs; however, no significant difference was found $(p=0.059)$. There was no significant difference when the first, second, and third UACR were directly compared in the differentiation of microalbuminuria. However, while the second uACR was more useful for differentiating microalbuminuria than the sum of proteinuria measured twice, the first and third uACR measurements did not differ to a statistically significant extent, with the sum of proteinuria measured twice. 


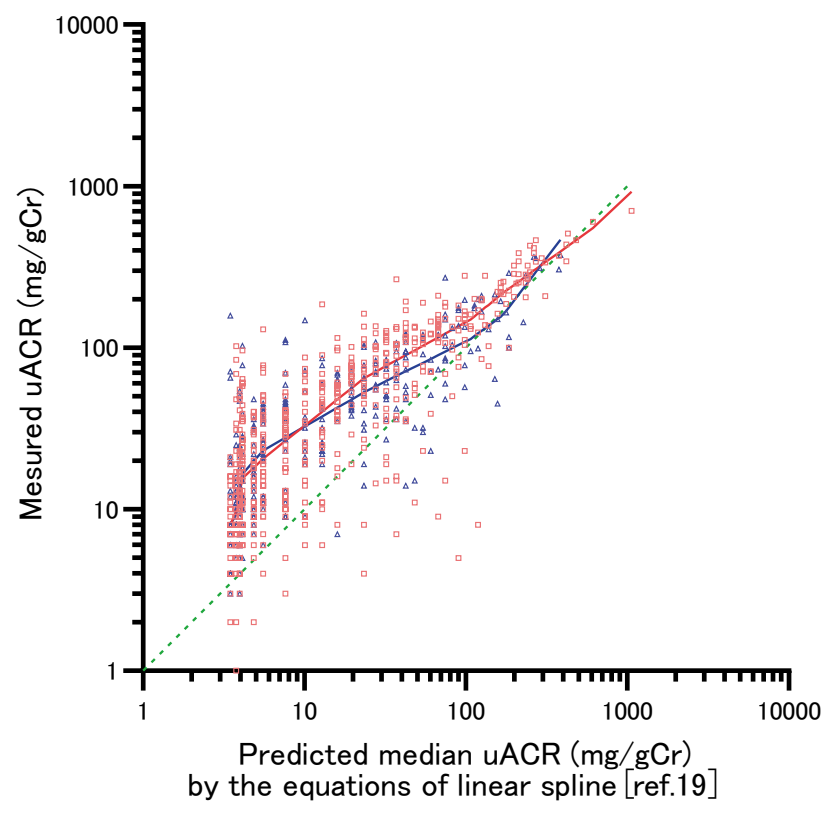

Fig. 5 The scatterplot of measured uACR in this study and predicted median UACR from the equations of linear spline in reference 19. The dotted line indicate the line of identity. Red curve indicates the predicted median uACR in non-diabetic patients and blue curve indicates the predicted median UACR in diabetic patients from linear spline model of this study. The measured uACRs are above the line of identity in the low predicted uACR in reference 19 . The predicted median uACRs in this study became approximately equal to the predicted value in reference 19 at higher levels of uACR. $\square$ : uACR of non-diabetic patients. $\triangle$ : uACR of diabetic patients

Factors affecting $\mathrm{CO}$ of the uPCR that differentiates a uACR of greater or less than $30 \mathrm{mg} / \mathrm{gCr}$, or microalbuminuria (Tables 8, 9).

When non-diabetics and diabetics were divided according to age, sex, or GFR category, the uPCR was useful for distinguishing between a uACR of greater or less than $30 \mathrm{mg} / \mathrm{gCr}$ or microalbuminuria in each respective division. While no effects of age or sex on the CO of uPCR were found in nondiabetics, the $\mathrm{CO}$ tended to be low in non-elderly people and in male diabetes patients. The effects of the GFR category were seen in non-diabetics and the $\mathrm{CO}$ in G1-3a tended to be lower than that in G3b-4.

The sensitivity, specificity and Youden's index for discriminating non-diabetic patients with a uACR of $\geq 30 \mathrm{mg}$ / $\mathrm{gCr}$ or micro- and macroalbuminuria by the uPCR according to GFR category are shown in Table 10. For uPCR 0.07, 0.09 and $0.15 \mathrm{~g} / \mathrm{gCr}$, the Youden's index of the $\mathrm{CO}$ for differentiating microalbuminuria was the largest at uPCR $0.09 \mathrm{~g} /$ $\mathrm{gCr}$ in $\mathrm{G} 1-4$ and G3b-4, and uPCR $0.07 \mathrm{~g} / \mathrm{gCr}$ in G1-3a.

When the $\mathrm{CO}$ that differentiated uACR $\geq 30 \mathrm{mg} / \mathrm{gCr}$ was reduced from uPCR $0.15 \mathrm{~g} / \mathrm{gCr}$ to $\mathrm{uPCR} 0.09 \mathrm{~g} / \mathrm{gCr}$, all 9 cases with a uACR of 100-299 mg/gCr in G1-3a and 8 of the 10 cases with a uACR of $100-299 \mathrm{mg} / \mathrm{gCr}$ in G3b-4 were newly determined as uACR $\geq 30 \mathrm{mg} / \mathrm{gCr}$ (Table 10). Out of 32 false positives associated with a CO of uPCR $0.07 \mathrm{~g} / \mathrm{gCr}$ in G1-3a, the uACR was $\geq 10 \mathrm{mg} / \mathrm{gCr}$ in 23 cases $(71.8 \%$ ), while all 11 false positives associated with a uPCR of $0.09 \mathrm{~g} /$ $\mathrm{gCr}$ in G3b-4 had a uACR of $\geq 10 \mathrm{mg} / \mathrm{gCr}$ (Table 11).

When examining the correlation of changes in the uACR and $\mathrm{uPCR}$, after dividing the first uPCR in non-diabetics and diabetics into greater than and less than $0.15 \mathrm{~g} / \mathrm{gCr}$, a positive correlation was confirmed with respect to the change of 2-1 times and 3-2 times when the first uPCR was less than and greater than $0.15 \mathrm{~g} / \mathrm{gCr}$ (Fig. 4).

Figure 5 indicates the predicted median ACR $(\mathrm{mg} / \mathrm{gCr})$ according to the equation from the linear spline from the uPCR by Weaver et al. [19] and the scatterplot of the measured ACR $(\mathrm{mg} / \mathrm{gCr})$ based on this study. The measured uACR did not distribute symmetrically along the dotted line of identity, with the low predicted median ACR distributed on the upper part of the dotted line. The red and blue lines indicate the predicted median ACR from the UPCR according to the linear spline in the non-diabetics and diabetics in this study, respectively. The predicted median uACR of $30 \mathrm{mg} / \mathrm{gCr}$, determined by the equation of Weaver et al. [19] corresponded to $74 \mathrm{mg} / \mathrm{gCr}$ for non-diabetics and $60 \mathrm{mg} /$ $\mathrm{gCr}$ for diabetics, as determined by the equation of this study, while the predicted mean uACR of $100 \mathrm{mg} / \mathrm{gCr}$ corresponded to $144 \mathrm{mg} / \mathrm{gCr}$ for non-diabetics and $109 \mathrm{mg} / \mathrm{gCr}$ for diabetics. Regarding the low predicted median uACR value, while the predicted median uACR value of this study was higher than that determined by the equation of Weaver et al., there was a tendency for the gap to become smaller as the UACR increased, with the values ultimately matching.

\section{The intraday and interday variance of UPCR and UACR of the same sample}

The study on the intra- and interday variance of uPCR and uACR included 88 subjects: age $73.1 \pm 12.7$ years (mean \pm SD); 48 males; and 40 females. Their underlying lifestyle-related disease was 65 cases of non-diabetic disease and 23 cases of diabetes. Regarding the $\mathrm{G}$ stage, there were 2 cases of G1, 18 cases of G2, 29 cases of G3a, 29 cases of $\mathrm{G} 3 \mathrm{~b}$, and 10 cases of $\mathrm{G} 4$.

The mean values of two urinary protein measurements were categorized into three groups: less than $3 \mathrm{mg} / \mathrm{dl}$ (hereinafter, the "low urinary protein concentration group"); $3 \mathrm{mg} / \mathrm{dl}$ or more but less than $8 \mathrm{mg} / \mathrm{dl}$ (hereinafter, the "medium urinary protein concentration group"); and $8 \mathrm{mg} /$ $\mathrm{dl}$ or more (hereinafter, the "high urinary protein concentration group") (Table 12, Fig. 6).

$\mathrm{The} \mathrm{uCr} / \mathrm{sCr}$ in the low urinary protein concentration group was lower than the high urinary protein concentration 


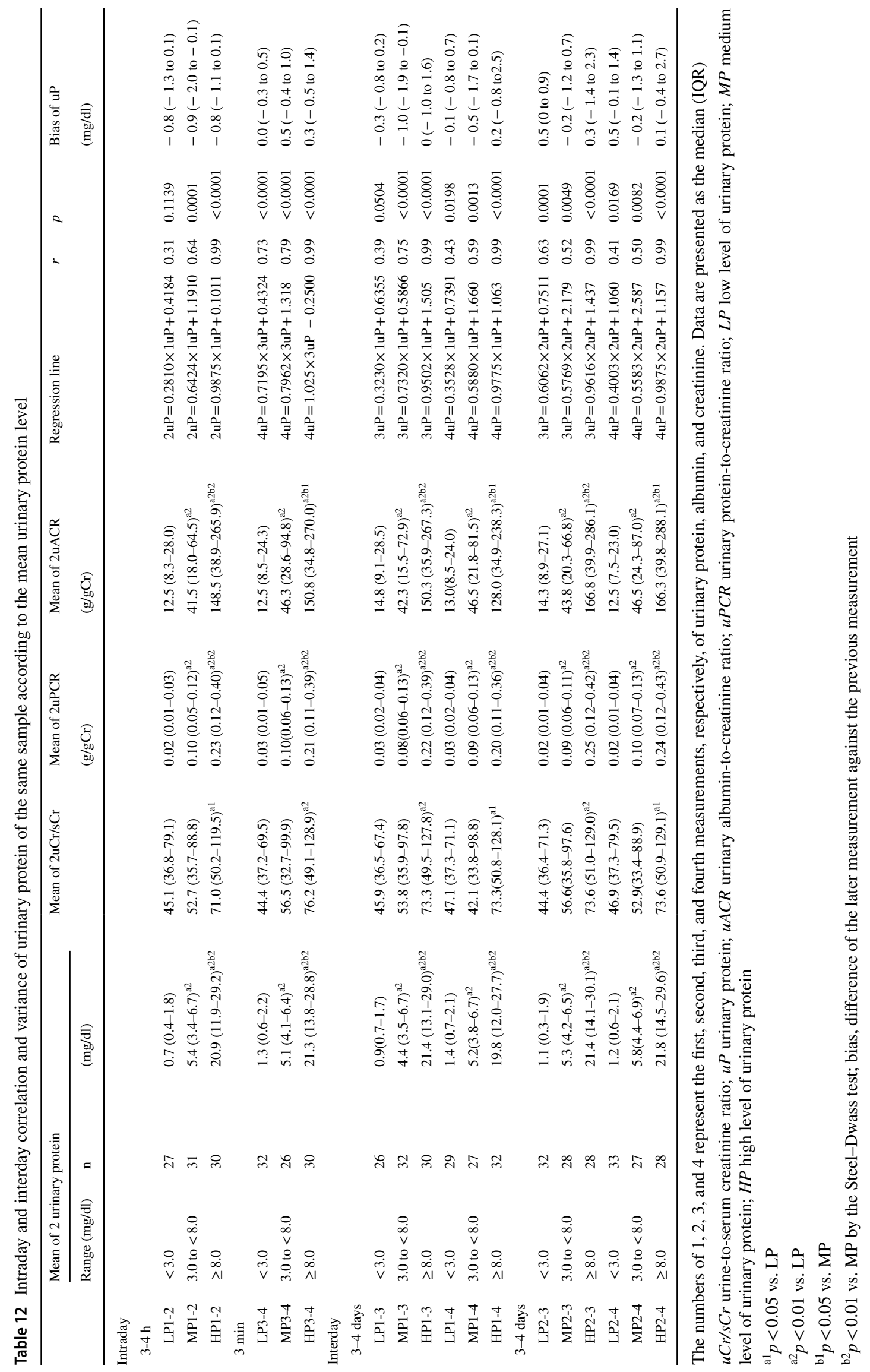


LP1-2

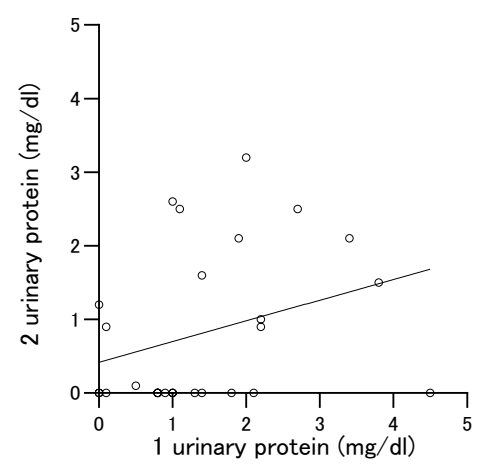

LA1-2

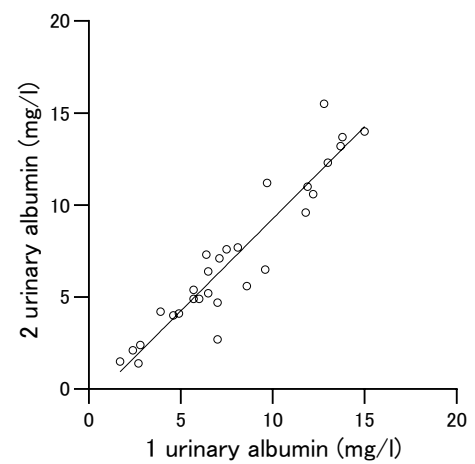

LP3-4

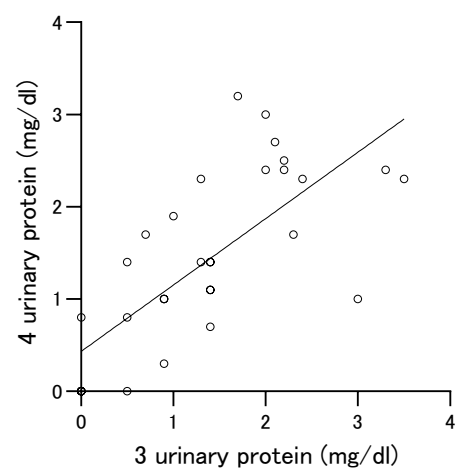

LA3-4

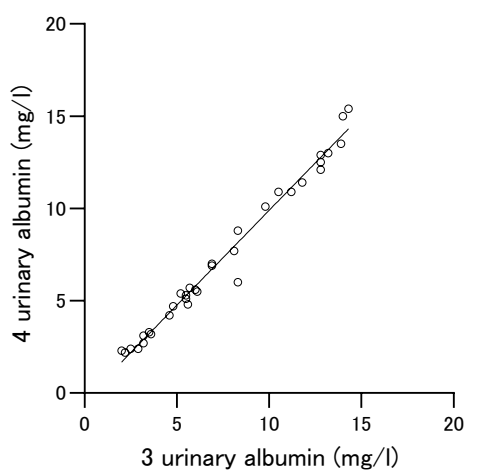

MP1-2

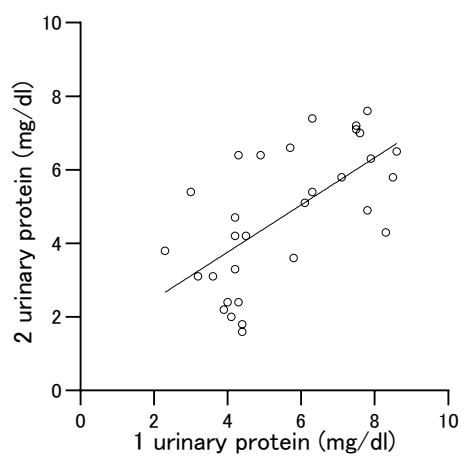

MA1-2

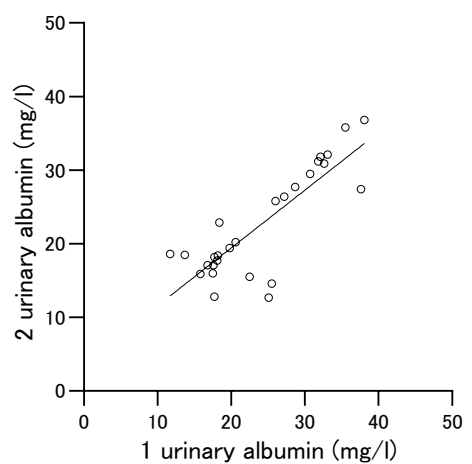

MP3-4

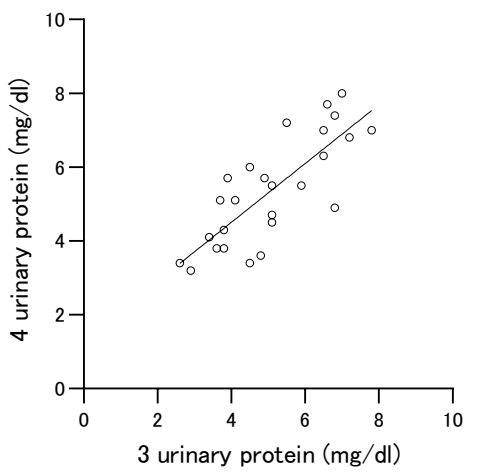

MA3-4

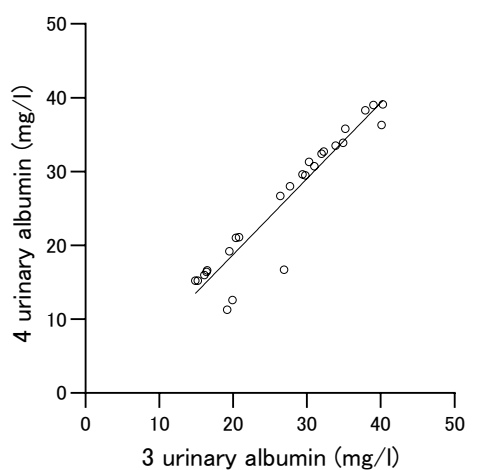

HP1-2

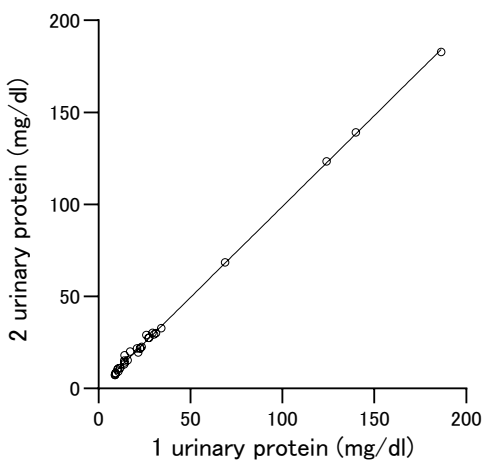

HA1-2

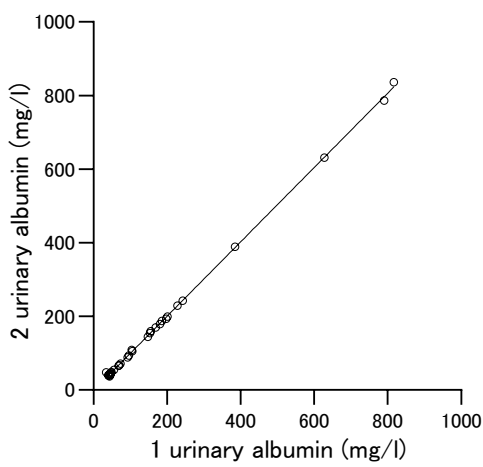

HP3-4

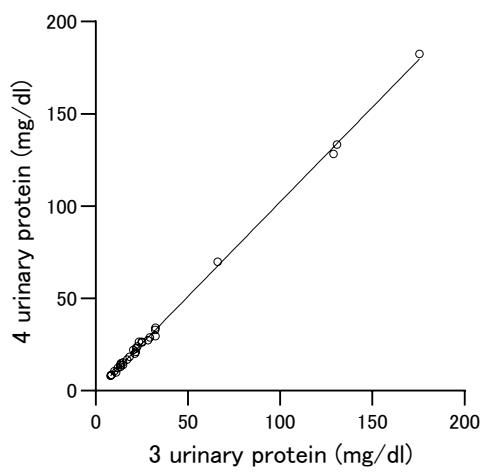

HA3-4

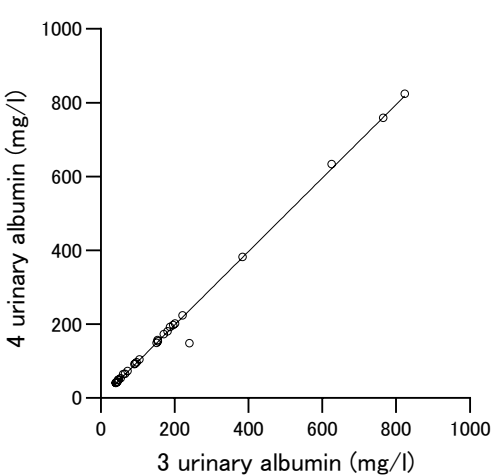


4Fig. 6 Intraday and interday correlation of urinary protein and albumin of the same sample according to the mean of each 2 levels. The symbols in the upper left of the figure correspond to the symbols in Tables 12, 13, 14. O, correlations between 1st and 2nd, 3rd and 4th, 1 st and 3rd, and 2nd and 3rd measurements; $\square$, correlations between 1 st and 4 th, and 2 nd and 4 th measurements

group $(p<0.05)$. Among the three groups, uPCR and uACR were significantly different. The median of uPCR and uACR was, respectively, 0.02 to $0.03 \mathrm{~g} / \mathrm{gCr}, 13-15 \mathrm{mg} / \mathrm{gCr}$ in the low urinary protein concentration group, $0.08-0.10 \mathrm{~g} / \mathrm{gCr}$, $42-47 \mathrm{mg} / \mathrm{gCr}$ in the medium urinary protein concentration group, and $0.21-0.25 \mathrm{~g} / \mathrm{gCr}, 128-166 \mathrm{mg} / \mathrm{gCr}$ in the high urinary protein concentration group (Table 12).

Regarding the intraday variance of urinary protein in the low urinary protein concentration group, no correlation was found between the first and second measurements with an interval of 3-4 h of refrigeration, while a significant positive correlation was noted between the third and fourth consecutive measurements 3-4 days later. Positive interday correlations were found between the first and fourth, second and third, and second and fourth measurements, but not between the first and third measurements. Both the intraday and interday correlations were positive and significant in the medium urinary protein concentration group. In the high urinary protein concentration group, the intraday and interday correlations were positive, significant, and even stronger.

The mean values of two measurements of albuminuria were categorized into three groups: less than $15 \mathrm{mg} / \mathrm{dl}$ (hereinafter, the "low urinary albumin concentration group"); $15 \mathrm{mg} / \mathrm{dl}$ or more but less than $40 \mathrm{mg} / \mathrm{dl}$ (hereinafter, the "medium urinary albumin concentration group"); and $40 \mathrm{mg} / \mathrm{dl}$ or more (hereinafter, the "high urinary albumin concentration group") (Table 13, Fig. 6). The uACR was also different among the three groups categorized according to the urinary albumin concentration. The median of uACR was $10-12 \mathrm{mg} / \mathrm{gCr}$ and $37-41 \mathrm{mg} / \mathrm{gCr}$ in the low and medium urinary albumin concentration groups, respectively (Table 13). The two intraday comparisons and four interday comparisons all indicated significant positive correlations in all groups categorized by albuminuria.

Urinary creatinine values were categorized into three groups: less than $50 \mathrm{mg} / \mathrm{dl} ; 50 \mathrm{mg} / \mathrm{dl}$ or more but less than $90 \mathrm{mg} / \mathrm{dl}$; and $90 \mathrm{mg} / \mathrm{dl}$ or more (Table 14). The two intraday comparisons and four interday comparisons all indicated considerable positive correlations in all groups.
We categorized the mean of uPCR values into two groups: less than $0.15 \mathrm{~g} / \mathrm{gCr}$ and $0.15 \mathrm{~g} / \mathrm{gCr}$ or more (Table 15, Fig. 7). For uPCR, the two intraday comparisons and four interday comparisons all indicated significant positive correlations in a both groups. For uACR, significant positive intraday and interday correlations were found in both groups.

The urinary protein concentration is useful to determine whether or not the $\mathrm{uACR}$ is $\geq 30 \mathrm{mg} / \mathrm{gCr}$ and the $\mathrm{CO}$ of urinary protein concentration was 3.3 to $4.0 \mathrm{mg} / \mathrm{dl}$ (Table 16). The CO value of uPCR to determine whether or not uACR is $\geq 30 \mathrm{mg} / \mathrm{gCr}$ was $0.08 \mathrm{~g} / \mathrm{gCr}$ in the the first, second, and fourth measurements, and $0.07 \mathrm{~g} / \mathrm{gCr}$ in the third measurement, each indicating a significantly higher ability to determine whether or not uACR is $\geq 30 \mathrm{mg} / \mathrm{gCr}$, compared to the urinary protein concentration measured simultaneously. The $\mathrm{CO}$ value of uPCR to determine whether or not the mean of the four uACR measurements is $\geq 30 \mathrm{mg} / \mathrm{gCr}$ was $0.08 \mathrm{~g} / \mathrm{gCr}$ in the first, second, and fourth measurements, and $0.07 \mathrm{~g} / \mathrm{gCr}$ in the third measurement (Table 17). There was no significant difference in the values of ROC curve area between the four measurements.

For the group in which the mean value of the two measurements of uPCR was $0.15 \mathrm{~g} / \mathrm{gCr}$ or more, both values were no less than the $\mathrm{CO}$ values, and in most cases, both of the two measurements resulted in a uACR $\geq 30 \mathrm{mg} / \mathrm{gCr}$ (Fig. 7). In the group of $\mathrm{uPCR}<0.15 \mathrm{~g} / \mathrm{gCr}$, the median uACR was 21 to $23 \mathrm{mg} / \mathrm{gCr}$, with the results indicating three different patterns: both measurements resulted in $\mathrm{uACR} \geq 30 \mathrm{mg} / \mathrm{gCr}$; only one measurement was uACR $\geq 30 \mathrm{mg} / \mathrm{gCr}$; and both measurements were uACR $<30 \mathrm{mg} / \mathrm{gCr}$. The median uPCR and $\mathrm{UACR}$ equivalent to a urinary protein concentration of less than $3 \mathrm{mg} / \mathrm{gCr}$ was 0.02 to $0.03 \mathrm{~g} / \mathrm{gCr}$ and 13 to $15 \mathrm{mg} /$ $\mathrm{gCr}$, respectively. The uPCR mostly fluctuated in a range less than the $\mathrm{CO}$ value.

In this study, the $\mathrm{CO}$ of urinary protein concentration that determines whether or not $\mathrm{uACR}$ is $\geq 30 \mathrm{mg} / \mathrm{gCr}$ in nondiabetic diabetes (591 times) and diabetes (318 times) was $7 \mathrm{mg} / \mathrm{dl}$ (AUC 0.749 SE 0.020 , sensitivity 0.67 , specificity $0.73, p<0.0001$ ) and $6 \mathrm{mg} / \mathrm{dl}$ (AUC 0.781 SE 0.026, sensitivity 0.70 , specificity $0.77, p<0.0001$ ), and the values of ROC curve area of urinary protein concentration were found to be significantly lower than those of uPCR $(p<0.0001)$, respectively. 
LP1-3, 4

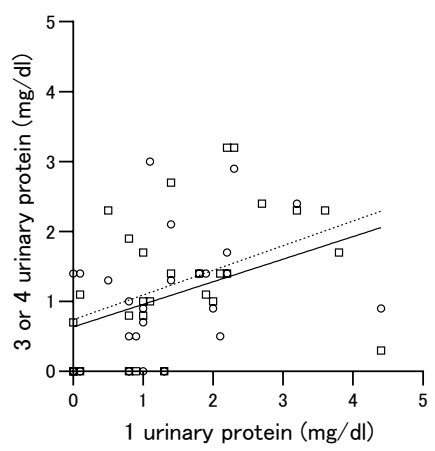

LA1-3, 4

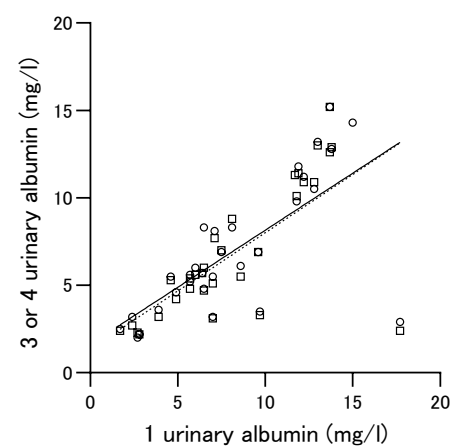

LP2-3, 4

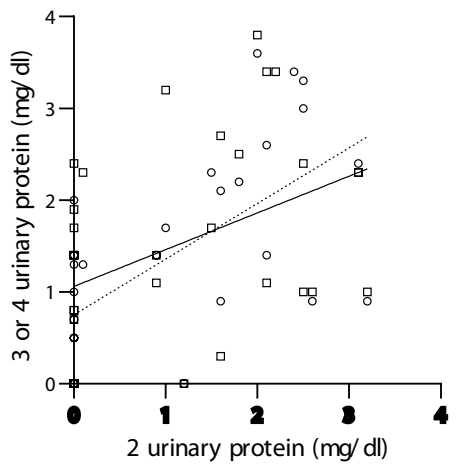

LA2-3, 4

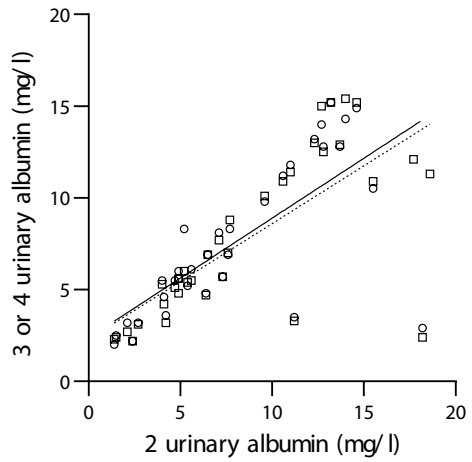

MP1-3, 4

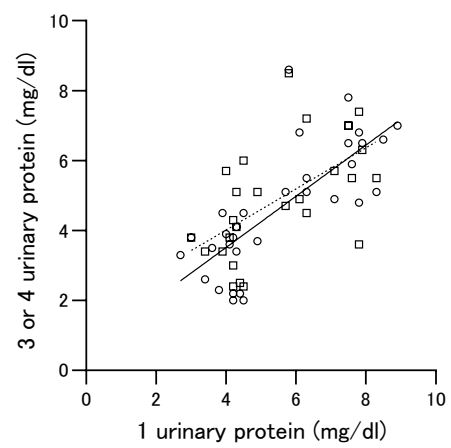

MA1-3, 4

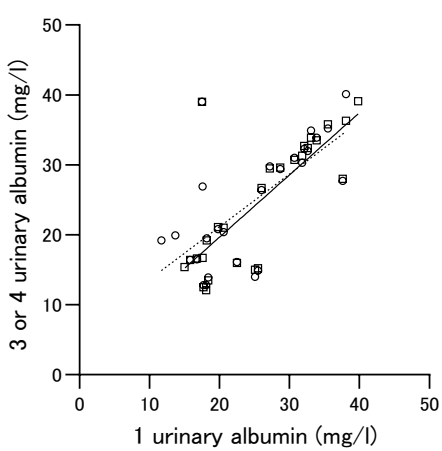

MP2-3, 4

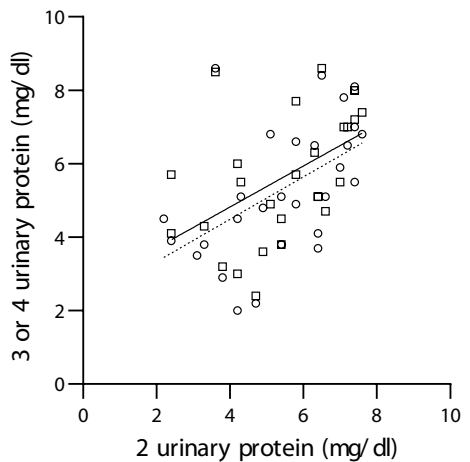

MA2-3, 4

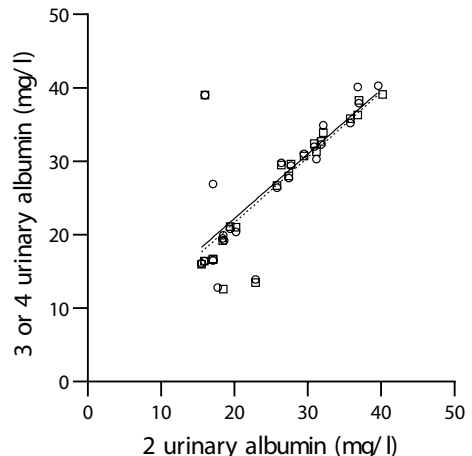

HP1-3, 4

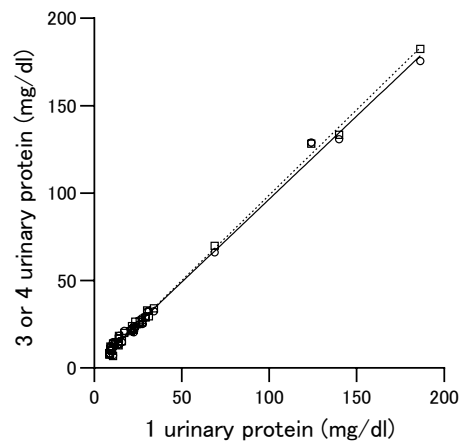

HA1-3, 4

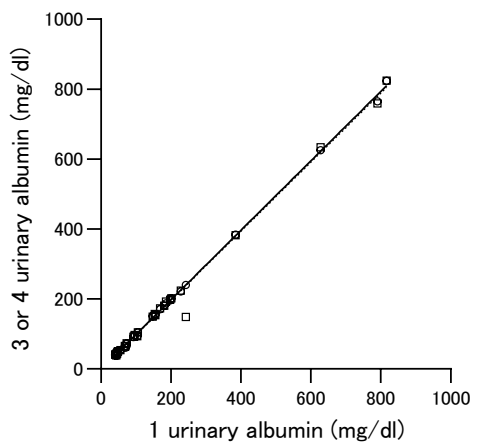

HP2-3, 4

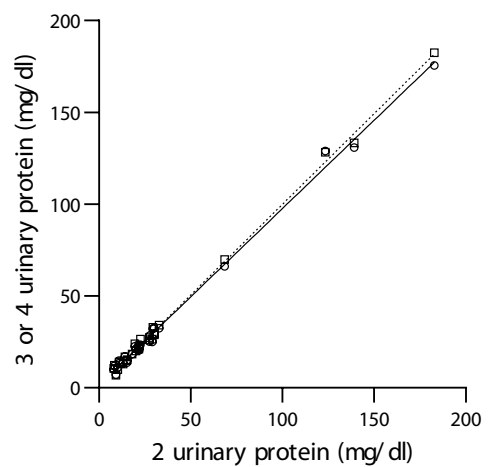

HA2-3, 4

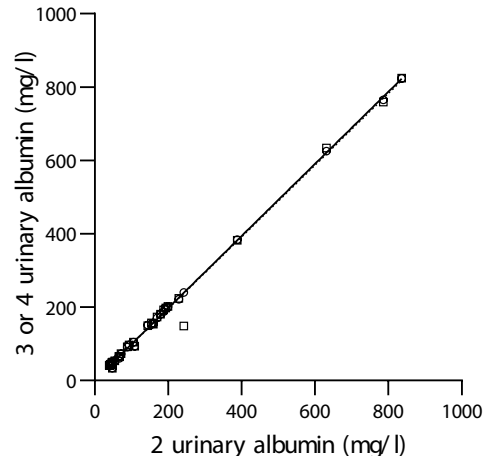

Fig. 6 (continued) 


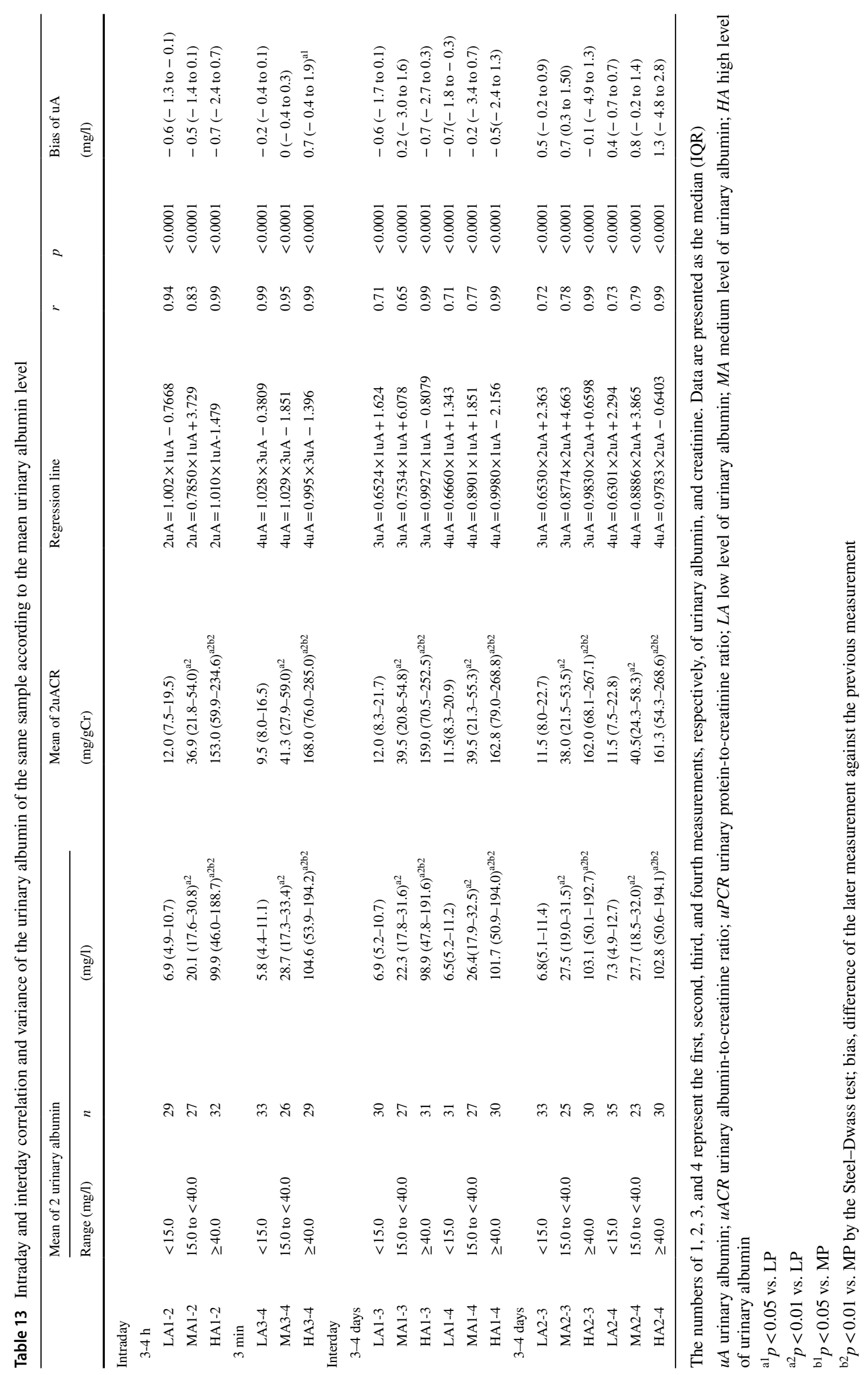


Table 14 Intraday and interday correlation and variance of urinary creatinine of the same sample according to the mean urinary creatinine level

\begin{tabular}{|c|c|c|c|c|c|c|c|}
\hline & \multicolumn{3}{|c|}{ Mean of 2 urinary creatinine } & \multirow[t]{2}{*}{ Regression line } & \multirow[t]{2}{*}{$r$} & \multirow[t]{2}{*}{$p$} & \multirow{2}{*}{$\begin{array}{l}\text { Bias of } \mathrm{uCr} \\
(\mathrm{mg} / \mathrm{dl})\end{array}$} \\
\hline & Range (mg/dl) & $n$ & $(\mathrm{mg} / \mathrm{dl})$ & & & & \\
\hline \multicolumn{8}{|l|}{ Intraday } \\
\hline \multicolumn{8}{|l|}{$3-4 \mathrm{~h}$} \\
\hline LC1-2 & $<50.0$ & 30 & $35.8(29.1-43.6)$ & $2 \mathrm{uCr}=1.001 \times 1 \mathrm{uCr}-0.06834$ & 0.99 & $<0.0001$ & $-0.1-0.2$ to 0.1$)$ \\
\hline Mcl-2 & 50.0 to $<90.0$ & 30 & $64.7(58.4-76.5)^{\mathrm{a} 2}$ & $2 \mathrm{uCr}=0.9962 \times 1 \mathrm{uCr}-0.2676$ & 0.99 & $<0.0001$ & $-0.1-0.4$ to 0.4$)$ \\
\hline $\mathrm{HC} 1-2$ & $\geq 90.0$ & 28 & $128.1(113.9-157.2)^{\mathrm{a} 2 \mathrm{~b} 2}$ & $2 \mathrm{uCr}=1.013 \times 1 \mathrm{uCr}-1.091$ & 0.99 & $<0.0001$ & $0.5(-0.6$ to 1.3$)$ \\
\hline \multicolumn{8}{|l|}{$3 \min$} \\
\hline LC3-4 & $<50.0$ & 30 & $35.9(28.7-43.7)$ & $4 \mathrm{uCr}=1.008 \times 3 \mathrm{uCr}+0.07271$ & 0.99 & $<0.0001$ & $0.1(-0.1$ to 0.4$)$ \\
\hline MC3-4 & 50.0 to $<90.0$ & 29 & $64.4(58.6-76.6)^{\mathrm{a} 2}$ & $4 \mathrm{uCr}=0.9911 \times 3 \mathrm{uCr}+0.6046$ & 0.99 & $<0.0001$ & $-0.1(-0.3$ to 0.3$)$ \\
\hline HC3-4 & $\geq 90.0$ & 29 & $126.9(114.5-156.0)^{\mathrm{a} 2 \mathrm{~b} 2}$ & $4 \mathrm{uCr}=0.9940 \times 3 \mathrm{uCr}+1.619$ & 0.99 & $<0.0001$ & $0.5(-0.4$ to 0.9$)$ \\
\hline \multicolumn{8}{|l|}{ Interday } \\
\hline \multicolumn{8}{|l|}{ 3-4 days } \\
\hline LC1-3 & $<50.0$ & 30 & $35.3(28.7-43.8)$ & $3 \mathrm{uCr}=0.9880 \times 1 \mathrm{uCr}+0.04098$ & 0.99 & $<0.0001$ & $-0.1(-0.2$ to 0.1$)$ \\
\hline MC1-3 & 50.0 to $<90.0$ & 29 & $64.5(58.5-76.7)^{\mathrm{a} 2}$ & $3 \mathrm{uCr}=1.023 \times 1 \mathrm{uCr}-1.095$ & 0.99 & $<0.0001$ & $0.3(-0.1-0.6)^{\mathrm{a} 1}$ \\
\hline $\mathrm{HC} 1-3$ & $\geq 90.0$ & 29 & $127.6(113.6-154.1)^{\mathrm{a} 2 \mathrm{~b} 2}$ & $3 \mathrm{uCr}=1.030 \times 1 \mathrm{uCr}-3.276$ & 0.99 & $<0.0001$ & $1.1(-0.5$ to 2.3$)$ \\
\hline LC1-4 & $<50.0$ & 30 & $36.0(29.0-43.8)$ & $4 \mathrm{uCr}=1.004 \times 1 \mathrm{uCr}-0.1729$ & 0.99 & $<0.0001$ & $0(-0.2$ to 0.4$)$ \\
\hline $\mathrm{MC} 1-4$ & 50.0 to $<90.0$ & 30 & $64.8(58.6-76.7)^{\mathrm{a} 2}$ & $4 \mathrm{uCr}=1.014 \times 1 \mathrm{uCr}-0.4915$ & 0.99 & $<0.0001$ & $0.3(-0.2$ to 1.0$)$ \\
\hline $\mathrm{HC} 1-4$ & $\geq 90.0$ & 28 & $128.0(114.2-157.6)^{\mathrm{a} 2 \mathrm{~b} 2}$ & $4 \mathrm{uCr}=1.023 \times 1 \mathrm{uCr}-1.646$ & 0.99 & $<0.0001$ & $1.1(-0.1$ to 2.4$)$ \\
\hline \multicolumn{8}{|l|}{ 3-4 days } \\
\hline LC2-3 & $<50.0$ & 30 & $35.3(28.8-43.6)$ & $3 \mathrm{uCr}=0.9859 \times 2 \mathrm{uCr}+0.1434$ & 0.99 & $<0.0001$ & $0(-0.4$ to 0.3$)$ \\
\hline MC2-3 & 50.0 to $<90.0$ & 29 & $64.6(58.3-76.4)^{\mathrm{a} 2}$ & $3 \mathrm{uCr}=1.022 \times 2 \mathrm{uCr}-1.109$ & 0.99 & $<0.0001$ & $0.5(0-0.7)^{\mathrm{a} 2}$ \\
\hline $\mathrm{HC} 2-3$ & $\geq 90.0$ & 29 & $127.7(114.0-154.6)^{\mathrm{a} 2 \mathrm{~b} 2}$ & $3 \mathrm{uCr}=1.016 \times 2 \mathrm{uCr}-2.048$ & 0.99 & $<0.0001$ & $0.6(-0.5$ to 1.4$)$ \\
\hline LC2-4 & $<50.0$ & 30 & $35.9(29.1-43.5)$ & $4 \mathrm{uCr}=1.002 \times 2 \mathrm{uCr}-0.09247$ & 0.99 & $<0.0001$ & $0.1(-0.1$ to 0.3$)$ \\
\hline MC2-4 & 50.0 to $<90.0$ & 30 & $64.9(58.5-76.5)^{\mathrm{a} 2}$ & $4 \mathrm{uCr}=1.017 \times 2 \mathrm{uCr}-0.7118$ & 0.99 & $<0.0001$ & $0.5(0.2-0.8)^{\mathrm{a} 2}$ \\
\hline $\mathrm{HC} 2-4$ & $\geq 90.0$ & 28 & $127.8(115.0-158.3)^{\mathrm{a} 2 \mathrm{~b} 2}$ & $4 \mathrm{uCr}=1.010 \times 2 \mathrm{uCr}-0.5403$ & 0.99 & $<0.0001$ & $0.8(-0.3$ to 1.7$)$ \\
\hline
\end{tabular}

The numbers of 1, 2, 3, and 4 represent the first, second, third, and fourth measurements, respectively, of urinary creatinine. Data are presented as median (IQR)

$u C r$ urinary creatinine, $L C$ low level of urinary creatinine; $M C$ medium level of urinary creatinine; $H C$ high level of urinary creatinine

${ }^{\mathrm{a} 1} p<0.05$ vs. LP

${ }^{\mathrm{a} 2} p<0.01$ vs. LP

${ }^{\mathrm{b} 1} p<0.05$ vs. MP

${ }^{\mathrm{b} 2} p<0.01$ vs. MP by the Steel-Dwass test; bias, difference of the later measurement against the previous measurement

\section{Discussion}

This study examined whether the uPCR could differentiate normoalbuminuria and microalbuminuria in adults with lifestyle-related diseases. This study targeted cases with uPCR of $<0.50 \mathrm{~g} / \mathrm{gCr}$. The target was set because the uACR was significantly beyond $30 \mathrm{mg} / \mathrm{gCr}$ for cases in which the uPCR increased by $>0.50 \mathrm{~g} / \mathrm{gCr}$ after the second measurement in this study, with a previous study reporting that there was a direct correlation between UACR and UPCR when uPCR was $>0.20 \mathrm{~g} / \mathrm{gCr}$ [17]. Although it is predicted that the sensitivity of microalbuminuria detected based on proteinuria would increase with the inclusion of cases in which the uPCR is $>0.50 \mathrm{~g} / \mathrm{gCr}$, it is believed that the $\mathrm{CO}$ value would not change since the value with the greatest Youden's index was selected as the $\mathrm{CO}$ value in this study. Moreover, it is believed that a CO of uPCR that can detect microalbuminuria with sufficient sensitivity for patients with little proteinuria is clinically necessary. It is also necessary to set a fixed value for the maximum limit of proteinuria when comparing the sensitivity to that in other groups.

Weaver et al.[19] examined the relationship between log (uACR) and log (uPCR), developed a formula for predicting the median uACR from the uPCR measured in the same day, and reported that the estimation was more accurate when the uPCR was more than $500 \mathrm{mg} / \mathrm{gCr}$. The range for predicting $\mathrm{UACR}$ from uPCR becomes wider in the low ranges of proteinuria. This is partly because the UPCR is less accurate than the $\mathrm{uACR}$ in lower ranges [22]. In addition, $\mathrm{uACR}$ and UPCR have different values depending on the damaged site of the kidney and the diversity of the disease state is greater in the low urinary protein ranges in individual cases 


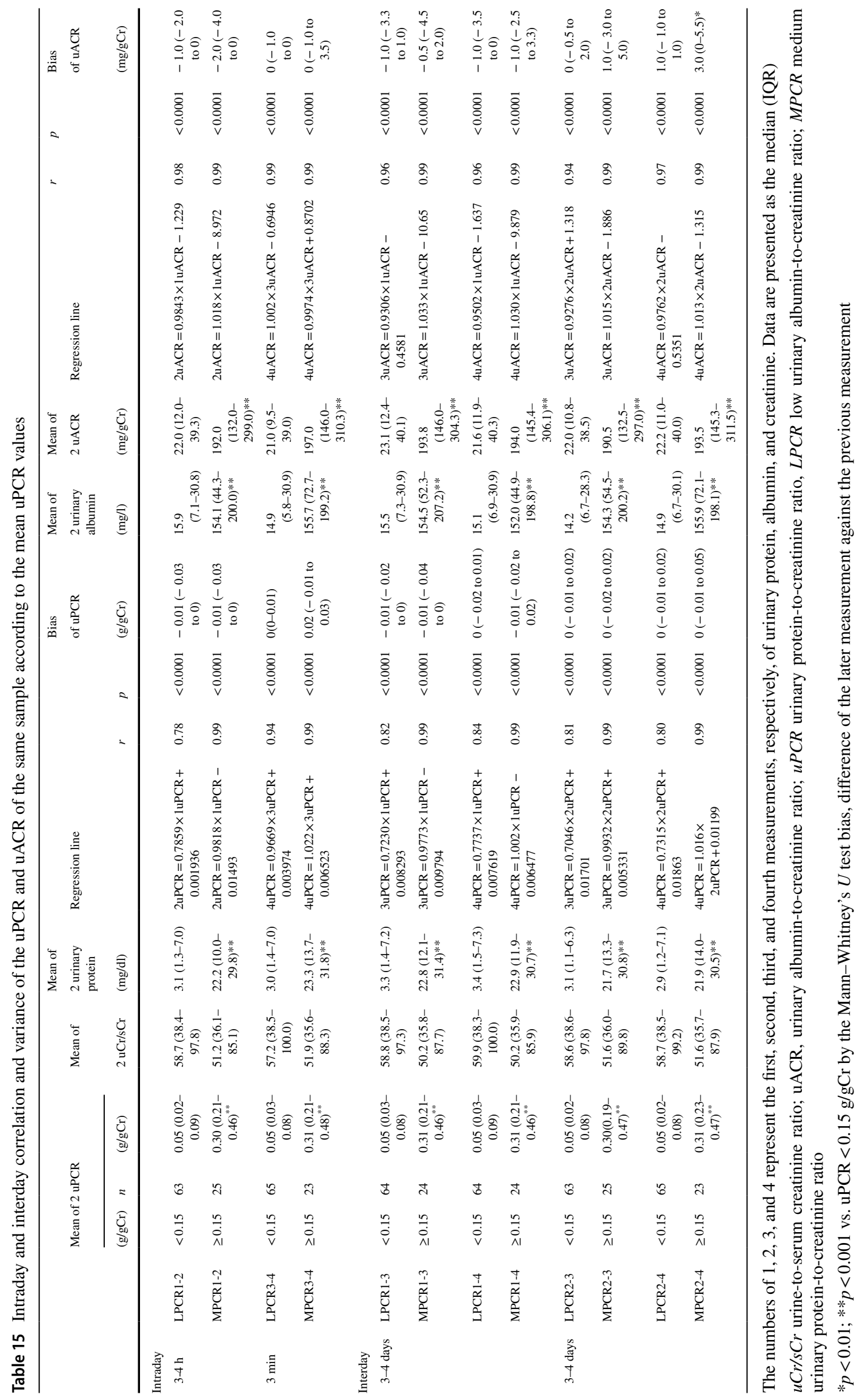


LPCR1-2

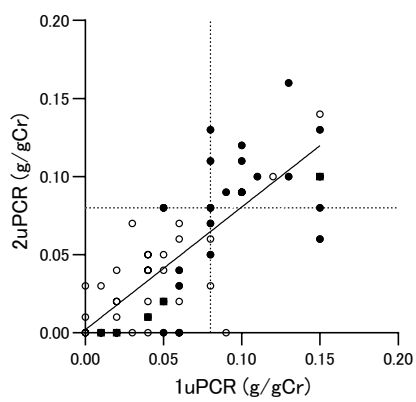

LPCR3-4

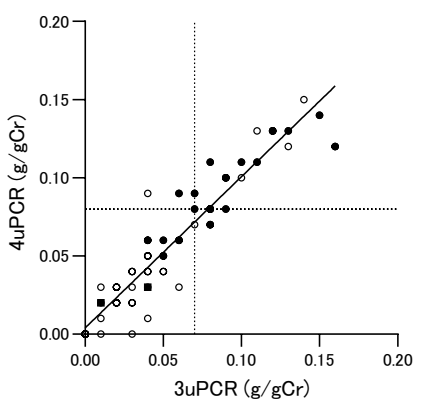

LPCR1-3

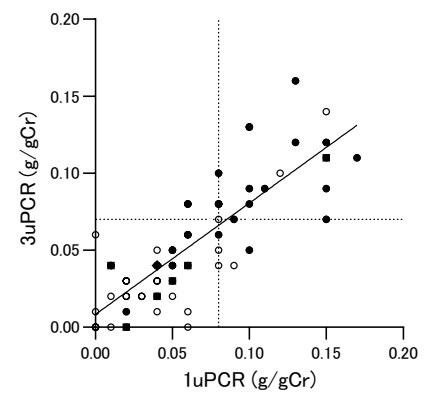

LPCR2-3

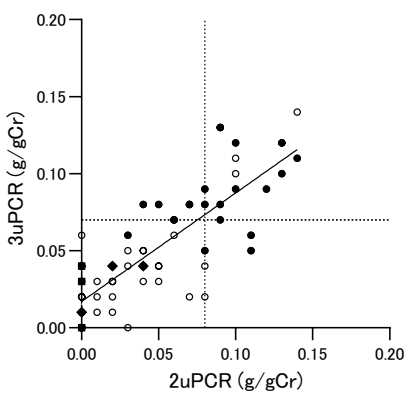

MPCR1-2

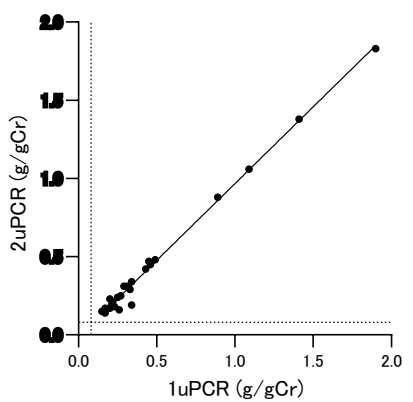

MPCR3-4

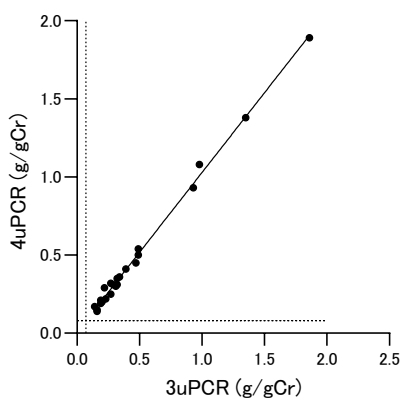

MPCR1-3

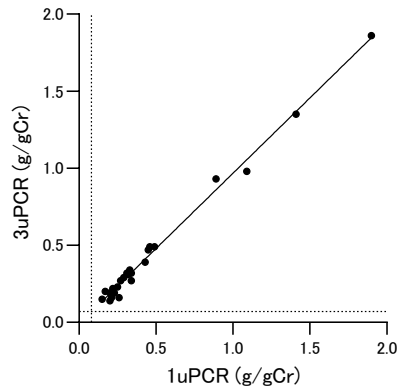

MPCR2-3

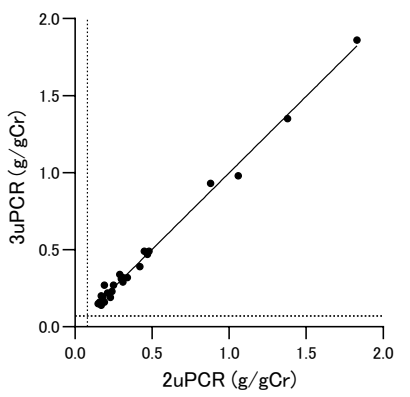

Fig. 7 Intraday and interday correlation of urinary protein creatinine ratio of the same sample according to the mean of 2 uPCR levels. The symbols in the upper left of the figure correspond to the symbols in Table 15. Both of the two measurements were uACR $30 \mathrm{mg} / \mathrm{gCr}$ or more; The previous measurement was $\mathrm{uACR} \geq 30 \mathrm{mg} / \mathrm{gCr}$, while
LPCR1-4

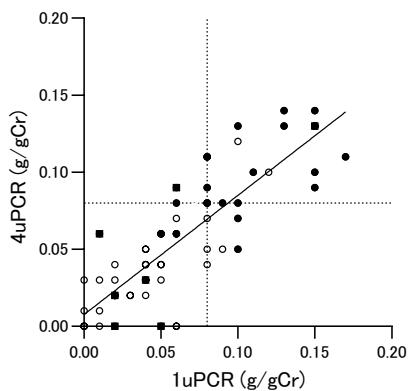

LPCR2-4

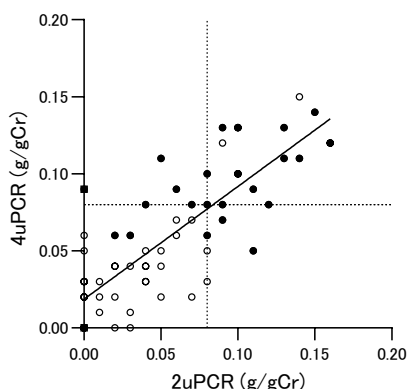

MPCR1-4

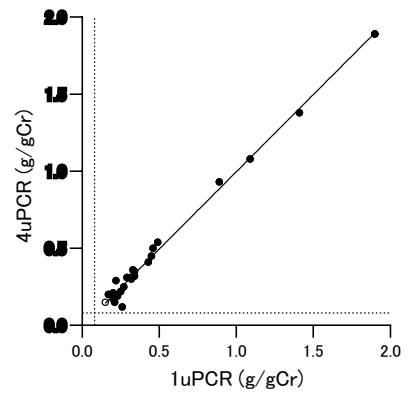

MPCR2-4

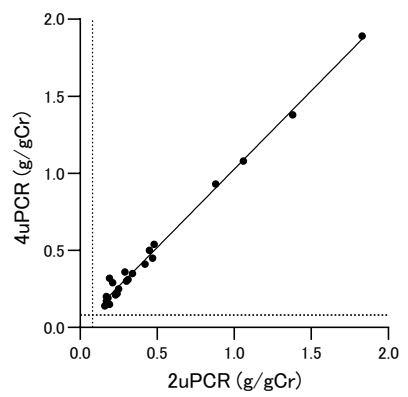

the later measurement was $\mathrm{uACR}<30 \mathrm{mg} / \mathrm{gCr}$;

The previous measurement was $\mathrm{uACR}<30 \mathrm{mg} / \mathrm{gCr}$, while the later measurement was uACR $\geq 30 \mathrm{mg} / \mathrm{gCr}$; $\bigcirc$ both measurements were uACR $<30 \mathrm{mg} /$ $\mathrm{gCr}$. The vertical and horizontal dotted lines indicate the $\mathrm{CO}$ value of $\mathrm{uPCR}$ to determine $\mathrm{uACR} \geq 30 \mathrm{mg} / \mathrm{gCr}$ for each measurement 
Table 16 Cut-off value (YI) of the urinary protein and $\mathrm{uPCR}$ to discriminate the samples of uACR $\geq 30 \mathrm{mg} / \mathrm{gCr}$

\begin{tabular}{lcccccccc}
\hline Test variables & $1 \mathrm{uP}$ & $2 \mathrm{uP}$ & $3 \mathrm{uP}$ & $4 \mathrm{uP}$ & $1 \mathrm{uPCR}$ & $2 \mathrm{uPCR}$ & $3 \mathrm{uPCR}$ & $4 \mathrm{uPCR}$ \\
\hline $\mathrm{uACR} \geq 30 \mathrm{mg} / \mathrm{gCr}$ & 52 & 47 & 47 & 45 & 52 & 47 & 47 \\
ROC curve area & 0.767 & 0.800 & 0.821 & 0.860 & $0.888^{\mathrm{a} 2}$ & $0.895^{\mathrm{a} 1}$ & $0.920^{\mathrm{a} 2}$ & $0.948^{\mathrm{a} 1}$ \\
Standard error & 0.051 & 0.047 & 0.045 & 0.040 & 0.034 & 0.036 & 0.029 & 0.022 \\
$p$ value & $<0.0001$ & $<0.0001$ & $<0.0001$ & $<0.0001$ & $<0.0001$ & $<0.0001$ & $<0.0001$ & $<0.0001$ \\
CO(YI) & 4.0 & 3.3 & 3.4 & 3.6 & 0.08 & 0.08 & 0.07 & 0.08 \\
Sensitivity & 0.85 & 0.85 & 0.89 & 0.91 & 0.81 & 0.83 & 0.85 & 0.89 \\
Specificity & 0.64 & 0.68 & 0.68 & 0.72 & 0.81 & 0.85 & 0.85 & 0.88 \\
\hline
\end{tabular}

The numbers of 1, 2, 3, and 4 represent the first, second, third, and fourth measurements, respectively, of urinary protein, albumin, and creatinine $C O(Y I)$ cut-off value using Youden's index, $u P$ urinary protein; $u A C R$ urinary albumin-to-creatinine ratio; $u P C R$ urinary protein-to-creatinine ratio

${ }^{\mathrm{a} 1} p<0.01$ vs. urinary protein

${ }^{\mathrm{a} 2} p<0.001$ vs. urinary protein measured at the same time

${ }^{\mathrm{a}}$ Unit of $\mathrm{uP}, \mathrm{mg} / \mathrm{dl}$; Unit of uPCR, g/gCr

Table 17 Comparison of cut-off value (YI) of the uPCR to discriminate the samples of mean of $4 \mathrm{uACR} \geq 30 \mathrm{mg} / \mathrm{gCr}$

\begin{tabular}{lllll}
\hline Test variables & 1uPCR & 2uPCR & 3uPCR & 4uPCR \\
\hline $\begin{array}{l}\text { Mean of } 4 \\
\text { uACR } \geq 30 \mathrm{mg} /\end{array}$ & 49 & 49 & 49 & 49 \\
$\quad \mathrm{gCr}$ & & & & \\
ROC curve area & 0.927 & 0.891 & 0.918 & 0.929 \\
Standard error & 0.026 & 0.036 & 0.029 & 0.029 \\
$p$ value & $<0.0001$ & $<0.0001$ & $<0.0001$ & $<0.0001$ \\
CO(YI) (g/gCr) & 0.08 & 0.08 & 0.07 & 0.08 \\
Sensitivity & 0.86 & 0.82 & 0.84 & 0.86 \\
Specificity & 0.82 & 0.87 & 0.87 & 0.92 \\
\hline
\end{tabular}

The numbers of 1, 2, 3, and 4 represent the first, second, third, and fourth measurements, respectively, of urinary protein, albumin, and creatinine

$C O(Y I)$ cut-off value using Youden's index; $u A C R$ urinary albuminto-creatinine ratio; $u P C R$ urinary protein-to-creatinine ratio

[19]. Although the ratio of the range of the first to third quartile for the median UACR in patients with low uPCR values was also wide, a relationship in which the median uACR increases as the uPCR increases was confirmed in the low ranges, with the slope of $\Delta \ln (\mathrm{uACR}) / \Delta \ln (\mathrm{uPCR})$ in the overall patients and in non-diabetic patients becoming steeper when the $\mathrm{uPCR}$ was $\geq 0.06 \mathrm{~g} / \mathrm{gCr}$. This time, we measured low levels of protein and examined whether it was possible to predict microalbuminuria.

The present study clarified that the uPCR was useful for differentiating $\mathrm{uACR} \geq 30 \mathrm{mg} / \mathrm{gCr}$ or microalbuminuria, and that the CO of the uPCR is $0.09 \mathrm{~g} / \mathrm{gCr}$. According to the equations by Weaver et al. [19], the uPCR corresponding to a median ACR of $30 \mathrm{mg} / \mathrm{gCr}$ is $0.139 \mathrm{~g} /$ $\mathrm{gCr}$, whereas the median ACR at uPCR $0.15 \mathrm{~g} / \mathrm{gCr}$ was $35.5 \mathrm{mg} / \mathrm{gCr}$ (IQR 16.0-65.8 mg/gCr). On the other hand, in the equation of this study, the uPCR corresponding to the median uACR of $30 \mathrm{mg} / \mathrm{gCr}$ was $0.077 \mathrm{~g} / \mathrm{gCr}$, whereas the median ACR corresponding to a uPCR of $0.15 \mathrm{~g} / \mathrm{gCr}$ was $78 \mathrm{mg} / \mathrm{gCr}$ in the overall patients. The predicted median uACR in this study was higher than the predicted value determined by the equation of Weaver et al. at low UPCR levels, with a tendency for the gap to become smaller and then to eventually match when the uPCR increased. Further studies are needed to determine the cause of the gap; however, it is believed that this is the reason why the $\mathrm{CO}$ of the uPCR which can differentiate microalbuminuria from normoalbuminuria is lower than $0.15 \mathrm{~g} / \mathrm{gCr}$, which is supposed to be equivalent to $\mathrm{uACR}$ $30 \mathrm{mg} / \mathrm{gCr}$ according to the KDIGO guideline [1].

The ability to differentiate microalbuminuria based on uPCR significantly increased with the sum of two or three of uPCRs in comparison to a single uPCR. Therefore, the sum of two or three consecutive uPCRs was considered useful for differentiating microalbuminuria.

With respect to the factors affecting $\mathrm{CO}$ in differentiating microalbuminuria, it has been reported that a higher uACR/ uPCR was associated with younger age [17-19], male sex $[18,19]$, non-white race [18], and diabetes mellitus [17-19]. This study indicated that age and sex did not affect the $\mathrm{CO}$ in non-diabetics. The difference in results may be attributed to the examination of the effects on the $\mathrm{CO}$ by dividing patients into non-diabetics and diabetics in this study.

In diabetics, the $\mathrm{CO}$ in males was lower than that in females and it is reported that males have a higher uACR/ $\mathrm{uPCR}$ ratio than females, with sex being the most important modifier of the relationship between UACR and UPCR [19]. However, since there were few patients with diabetes in this study, further examination of the effect of sex is believed to be necessary. 
With respect to the effect of the GFR category on CO in differentiating microalbuminuria, the $\mathrm{CO}$ tended to be higher due to the progression of the GFR category, which was $0.07 \mathrm{~g} / \mathrm{Cr}$ in G1-3a and $0.10 \mathrm{~g} / \mathrm{g} \mathrm{Cr}$ in G3b-4 in nondiabetics. It has been reported that the uACR corresponding to a uPCR of $0.15 \mathrm{~g} / \mathrm{gCr}$ is lower in G4 and G5 in comparison to eGFR $>30 \mathrm{ml} / \mathrm{min} / 1.73 \mathrm{~m}^{2}$ [19]. The reason for the increase in the $\mathrm{CO}$ may be attributed to a decrease in urinary protein selectivity, tubular proteinuria due to the progression of interstitial lesions, or the possible increase in urinary protein components, other than albumin, due to differences in protein reabsorption in the renal tubules [23]. On the other hand, it has also been reported that the ratio of albumin in the urinary protein increases as the GFR decreases $[17,18]$. However, because the ratio of albumin in the urinary protein increases with urinary albumin levels of up to $500 \mathrm{mg} / \mathrm{gCr}$ and urinary protein levels of up to $1000 \mathrm{mg} / \mathrm{gCr}$ [17], the studies that included overt proteinuria may have been looking at the effect of urinary protein, which increased as the GFR category progressed.

As for the clinical application for uPCR to uACR conversion equations, Weaver et al. [19] recommend measuring the uACR when possible, and estimating the median as well as the 25th and 75th percentiles of the ACR, if it is not feasible. However, the measurement of the UACR in non-diabetic patients has not been given insurance coverage in Japan. By differentiating microalbuminuria with uPCR of $0.09 \mathrm{~g} / \mathrm{gCr}$ in G1-4 or $0.07 \mathrm{~g} / \mathrm{gCr}$ in G1-3a-rather than a uPCR of $0.15 \mathrm{~g} /$ $\mathrm{gCr}$-although the specificity becomes lower, the sensitivity in detecting microalbuminuria increases. In particular, it becomes possible to detect microalbuminuria of $>100 \mathrm{mg} /$ $\mathrm{gCr}$, which is a relatively high level. This suggests that the clinical application of the prediction of the UACR from uPCR may potentially enable early intervention for patients with microalbuminuria.

On the other hand, when microalbuminuria was differentiated by uPCR of 0.09 or $0.07 \mathrm{~g} / \mathrm{gCr}$, false positive findings were detected more frequently in comparison to when microalbuminuria was differentiated by a uPCR of $0.15 \mathrm{~g} /$ $\mathrm{gCr}$. However, false positive findings were more frequently observed in cases with a uACR of $\geq 10 \mathrm{mg} / \mathrm{gCr}$ in comparison to those with a uACR of $<10 \mathrm{mg} / \mathrm{gCr}$. It is reported that the risk of cardiovascular disease and death increases at uACR levels of $<10 \mathrm{mg} / \mathrm{gCr}$ or albuminuria $10 \mathrm{mg} /$ day $[5,6,10]$, and a very low level of microalbuminuria is also a risk factor, independent of the kidney function, and the presence of hypertension and diabetes [6]. This suggests that the disadvantages of an increased false positive rate may be relatively low.

This study indicated the overall high degree of positive correlation between changes in the uPCR and uACR in patients with an initial uPCR $<0.15$ or $\geq 0.15 \mathrm{~g} / \mathrm{gCr}$, suggesting that the UPCR may be useful for monitoring the course of microalbuminuria. This may be the reason why the ability to differentiate microalbuminuria is increased by the sum of multiple uPCR values in non-diabetic patients.

Among patients with non-diabetic lifestyle-related diseases, microalbuminuria was confirmed in $42.7 \%$ of cases that were negative proteinuria by dipstick analysis on the first measurement, $76.7 \%$ of the ( \pm ) cases, $81.0 \%$ of the $(1+)$, and all $(2+)$ cases. Normally, lifestyle improvement and health guidance are provided when proteinuria is $( \pm)$ or higher at a checkup. In the event that proteinuria ( \pm ) persists, it is necessary to visit a medical institution. Since outpatients were targeted and the frequency of microalbuminuria was high despite proteinuria negativity in this study, it is believed that an early diagnosis and intervention for microalbuminuria in non-diabetic lifestyle-related diseases may be possible by determining the quantity of proteinuria, even it is only measured once while the patient is negative for proteinuria by dipstick analysis. While the sum of multiple measurements of urinary protein is useful for diagnosing microalbuminuria, it is desirable to make a tentative diagnosis after the first measurement to ensure that patients continue to visit the hospital. It is therefore necessary to evaluate the usefulness of multiple measurements including the cost-benefit.

The intra- and interday variance of uPCR and uACR of the same sample is influenced by urinary protein and urinary albumin concentrations, as the urinary creatinine has a considerable positive correlation and is nearly the same. In the group with urinary protein less than $3 \mathrm{mg} / \mathrm{dl}$, the consecutive measurements indicated significant positive correlations. However, the measurements after several hours of refrigeration, several days of freezing, and thawing had insignificant or reduced correlations, indicating that specimen preservation may have influenced the measurement of traces of protein. In the group with a urinary protein concentration of $3 \mathrm{mg} / \mathrm{dl}$ or more, there was a significant positive interday and intraday correlation, which was reproducible. In the group with a low urinary albumin concentration of less than $15 \mathrm{mg} / \mathrm{l}$, the median uACR was equivalent to that of normoalbuminuria, wherein the measurements had an intraday and interday positive correlation, which was relatively stable and reproducible.

The CO of uPCR to determine whether uACR is $30 \mathrm{mg} /$ $\mathrm{gCr}$ or more was 0.07 to $0.08 \mathrm{~g} / \mathrm{gCr}$ and underwent no intraday or interday changes. The reason for this is that when uPCR is $>0.15 \mathrm{~g} / \mathrm{gCr}$, in most cases, $\mathrm{uACR}$ is $\geq 30 \mathrm{mg} / \mathrm{gCr}$, so the $\mathrm{CO}$ value mainly depends on the relation between the two values when $\mathrm{uPCR}$ is less than $0.15 \mathrm{~g} / \mathrm{gCr}$. When $\mathrm{uPCR}$ is less than $0.15 \mathrm{~g} / \mathrm{gCr}$, the median of urinary albumin was approximately $15 \mathrm{mg} / \mathrm{l}$ and $\mathrm{uACR}$ had a positive correlation. On the other hand, uPCR also had significant positive correlations as it includes cases with proteinuria of $\geq 3 \mathrm{mg} /$ dl. When the urinary protein concentration is low, at less than $3 \mathrm{mg} / \mathrm{dl}$, the correlation of uPCR is also expected to be 
poor; however, the median uPCR is low at 0.02 to $0.03 \mathrm{~g} /$ $\mathrm{gCr}$, the median uACR corresponds to normoalbuminuria, and UPCR fluctuates within the range less than the $\mathrm{CO}$ to determine the microalbuminuria. Therefore, it is believed that the poor correlation of low urinary protein concentrations did not affect the $\mathrm{CO}$ value and discrimination ability of microalbuminuria.

In this study, the urinary protein was rapidly measured using morning urine and it was found that the $\mathrm{CO}$ of urinary protein concentration to determine whether $\mathrm{UACR}$ is $30 \mathrm{mg} /$ $\mathrm{gCr}$ or more was $7 \mathrm{mg} / \mathrm{dl}$ for non-diabetes and $6 \mathrm{mg} / \mathrm{dl}$ for diabetes, respectively. Since they were $3 \mathrm{mg} / \mathrm{dl}$ or more, it is predicted that the $\mathrm{CO}$ value of UPCR to determine the microalbuminuria did not change even if measured the same day after refrigeration or after several days of cryopreservation. The CO of uPCR to discriminate microalbuminuria should be evaluated using uPCR because the urinary protein concentration depends on the urine dilution status. Although the urinary protein concentration should be considered in terms of the reproducibility of UPCR values in preserved specimens, the poor correlation of low urinary protein concentration has less impact on CO of UPCR in determining microalbuminuria with the sufficient urinary creatinine concentration, as the uPCR only fluctuates within the range below the $\mathrm{CO}$.

The present study was associated with some limitations. Because the sample collection time and measurement/analysis method differed at each facility, the relationship between the uACR and uPCR may differ, making it necessary to set the $\mathrm{CO}$ of the uPCR for distinguishing microalbuminuria at each facility.

In conclusion, the uPCR may be useful for predicting microalbuminuria in non-diabetic adults with lifestylerelated diseases. In this study, the CO of the uPCR that predicted microalbuminuria in non-diabetic lifestyle-related diseases was $0.09 \mathrm{~g} / \mathrm{gCr}$ for the GFR category of G1-4 and $0.07 \mathrm{~g} / \mathrm{gCr}$ for the GFR category of G1-3a. It is anticipated that kidney failure and cardiovascular disease will be reduced by strengthening intervention for CKD due to nondiabetic lifestyle-related diseases from the early stages.

Acknowledgement The authors would like to thank the staff of the Department of Internal Medicine and Mr. Junya Tasaki, Mr. Kazuyoshi Nogi, and other members of Clinical Laboratory for their cooperation in this research.

\section{Compliance with ethical standards}

Conflict of interest The authors have declared that no conflict of interest exists.

Human and animal rights All procedures performed in studies involving human participants were in accordance with the ethical standards of the institutional review board at Yuurinkouseikai Fuji Hospital and with the 1964 Helsinki declaration and its later amendments or compa- rable ethical standards. IRB approval has been obtained (IRB approval number 201709-3).

Informed consent Informed written consent was obtained from all individual participants included in the study.

Open Access This article is licensed under a Creative Commons Attribution 4.0 International License, which permits use, sharing, adaptation, distribution and reproduction in any medium or format, as long as you give appropriate credit to the original author(s) and the source, provide a link to the Creative Commons licence, and indicate if changes were made. The images or other third party material in this article are included in the article's Creative Commons licence, unless indicated otherwise in a credit line to the material. If material is not included in the article's Creative Commons licence and your intended use is not permitted by statutory regulation or exceeds the permitted use, you will need to obtain permission directly from the copyright holder. To view a copy of this licence, visit http://creativecommons.org/licenses/by/4.0/.

\section{References}

1. Kidney Disease: Improving Global Outcomes. Clinical practice guideline for the evaluation and management of chronic kidney disease. Kidney Int Suppl. 2012;2013(3):19-90.

2. Japanease Society of Nephrology. Evidence-based Clinical Practice Guideline for CKD 2013. Clin Exp Nephrol. 2014; 18:346-423.

3. Nitta K, Masakane I, Hanafusa N, Taniguchi M, Hasegawa T, Nakai S, Goto S, Wada A, Hamano T, Hoshino J, Joki N, Abe M, Yamamoto K, Nakamoto H, Japanese Society for Dialysis Therapy Renal Data Registry Committee. Annual dialysis data report 2017, JSDT Renal data registry. Renal Replace Ther. 2019. https://doi. org/10.1186/s41100-019-0248-1.

4. Wada T, Haneda M, Furuichi K, Babazono T, Yokoyama H, Iseki K, Araki S, Ninomiya T, Hara S, Suzuki Y, Iwano M, Kusano E, Moriya T, Satoh H, Nakamura H, Shimizu M, Toyama T, Hara A, Makino H, The research group of diabetic nephropathy, Ministry of Health, Labour, and Welfare of Japan. Clinical impact of albuminuria and glomerular filtration rate on renal and cardiovascular events, and all-cause mortality in Japanese patients with type 2 diabetes. Clin Exp Nephrol. 2014;18:613-20.

5. Gerstein HC, Mann JF, Yi Q, Zinman B, Dinneen SF, Hoogwerf B, Halle JP, Young J, Rashkow A, Joyce C, Nawaz S, Yusuf S. Albuminuria and risk of cardiovascular events, death, and heart failure in diabetics and nondiabetic individuals. JAMA. 2001;286:421-6.

6. Klausen K, Borch-Johnsen K, Feldt-Rasmussen B, Jensen G, Clausen P, Scharling H, Appleyard M, Jensen JS. Very low levels of microalbuminuria are associated with increased risk of coronary heart disease and death independently of renal function, hypertension, and diabetes. Circulation. 2004;2004(110):32-5.

7. Weir MR. Microalbuminuria and cardiovascular disease. Clin J Am Soc Nephrol. 2007;2:581-90.

8. Segura J, Campo C, Ruilope LM. Effect of proteinuria and glomerular filtration rate on cardiovascular risk in essential hypertension. Kidney Int Suppl. 2004;92:S45-9.

9. van der Velde M, Bello AK, Brantama AH, EI Nahas M, Bakker SJ, de Jong PE, et al. Do albuminuria and hs-CRP add to the International Diabetes Federation definition of the metabolic syndrome in predicting outcome? Nephrol Dial Transplant. 2012;27:2275-83. 
10. Arnlov J, Evans JC, Meigs JB, Wang TJ, Fox CS, Levy D, Benjamin EJ, D'Agostino RB, Vasan RS. Low-grade albuminuria and incidence of cardiovascular disease events in nonhypertensive and nondiabetic individuals: the Framingham Heart Study. Circulation. 2005;112:969-75.

11. Takae K, Hata J, Ohara T, Yoshida D, Shibata M, Mukai N, Hirakawa Y, Kishimoto H, Tsuruya K, Kitazono K, Kiyohara Y, Ninomiya T. Albuminuria increases the risks for both Alzheimer Disease and Vascular Dementia in Community-Dwelling Japanease Elderly: the Hisayama Study. J Am Heart Assoc. 2018. https ://doi.org/10.1161/JAHA.117.006693.

12. Iseki $\mathrm{K}$, Ikemiya $\mathrm{Y}$, Iseki $\mathrm{C}$, Takishita S. Proteinuria and the risk of developing end-stage renal disease. Kidney Int. 2003;63:1468-74.

13. Sato H, Konta T, Ichikawa K, Suzuki N, Kabasawa A, Suzuki $\mathrm{K}$, et al. Comparison of the predictive ability of albuminuria and dipstick proteinuria for mortality in the Japanese population: the Yamagata(Takahata) study. Clin Exp Nephrol. 2016;20:611-7.

14. Nagai K, Yamagata K. Quantitative evaluation of proteinuria for health checkups is more efficient than the dipstick method. Clin Exp Nephrol. 2015;19:152-3.

15. Collier G, Greenan MC, Brady JJ, Murray B, Cunningham SK. A study of the relationship between albuminuria, proteinuria and urinary reagent strips. Ann Clin Biochem. 2009;46:247-9.

16. Methven S, MacGregor MS, Traynor JP, O'Reilly DS, Deighan CJ. Assessing proteinuria in chronic kidney disease: protein-creatinine ratio versus albumin-creatinine ratio. Nephrol Dial Transplant. 2010;25:2991-6.

17. Wu MT, Lam KK, Lee WC, Hsu KT, Wu CH, Cheng BC, Ng HY, Chi PJ, Lee YT, Lee CT. Albuminuria, proteinuria, and urinary albumin to protein ratio in chronic kidney disease. J Clin Lab Anal. 2012;26:82-92.

18. Fisher H, Hsu CY, Vittinghoff E, Lin F, Bansal N. Comparison of associations of urine protein-creatinine ratio versus albumin-creatinine ratio with complication of CKD: a cross-sectional analysis. Am J Kidney Dis. 2013;62:1102-8.

19. Weaver RG, James MT, Ravani P, Weaver CGW, Lamb EJ, Tonelli M, Manns BJ, Quinn RR, Jun M, Hemmelgarn BR. Estimating urine albumin-to-creatinine ratio from protein-to-creatinine ratio: development of equations using same-day measurements. J Am Soc Nephrol. 2020;31:591-601.

20. Fuhrman DY, Schneider MF, Dell KM, Blydt-Hansen TD, Mak R, Saland JM, Furth SL, Warady BA, Moxey-Mims MM, Schwartz GJ. Alubuminuria, proteinuria, and renal disease progression in children with CKD. Clin J Am Soc Nephrol. 2017;12:912-20.

21. Harrel FE. Regression modeling strategies: with applications to linear models, logistic and ordinal regression, and survival analysis. Berlin: Springer International Publishing; 2020.

22. Lamb EJ, MacKenzie F, Stevens PE. How should proteinuria be detected and measured. Ann Clin Biochem. 2009;46:215-7.

23. ChristenEI NR, Birn H. Renal filtration, transport, and metabolism of albumin and albuminuria. In: Alpen RJ, Caplan MJ, Moe O, editors. Seldin and Giebischs the kidney physiology and pathophysiology. Amsterdam: Elsevier; 2013. p. 2457-74.

Publisher's Note Springer Nature remains neutral with regard to jurisdictional claims in published maps and institutional affiliations. 